\title{
Physics of Three-Dimensional Bosonic Topological Insulators: Surface-Deconfined Criticality and Quantized Magnetoelectric Effect
}

\author{
Ashvin Vishwanath \\ Department of Physics, University of California, Berkeley, California 94720, USA \\ Materials Science Division, Lawrence Berkeley National Laboratories, Berkeley, California 94720, USA
}

\section{T. Senthil}

Department of Physics, Massachusetts Institute of Technology, Cambridge, Massachusetts 02139, USA (Received 4 October 2012; revised manuscript received 31 December 2012; published 28 February 2013)

\begin{abstract}
We discuss physical properties of "integer" topological phases of bosons in $D=3+1$ dimensions, protected by internal symmetries like time reversal and/or charge conservation. These phases invoke interactions in a fundamental way but do not possess topological order; they are bosonic analogs of freefermion topological insulators and superconductors. While a formal cohomology-based classification of such states was recently discovered, their physical properties remain mysterious. Here, we develop a field-theoretic description of several of these states and show that they possess unusual surface states, which, if gapped, must either break the underlying symmetry or develop topological order. In the latter case, symmetries are implemented in a way that is forbidden in a strictly two-dimensional theory. While these phases are the usual fate of the surface states, exotic gapless states can also be realized. For example, tuning parameters can naturally lead to a deconfined quantum critical point or, in other situations, to a fully symmetric vortex metal phase. We discuss cases where the topological phases are characterized by a quantized magnetoelectric response $\theta$, which, somewhat surprisingly, is an odd multiple of $2 \pi$. Two different surface theories are shown to capture these phenomena: The first is a nonlinear sigma model with a topological term. The second invokes vortices on the surface that transform under a projective representation of the symmetry group. We identify a bulk-field theory consistent with these properties, which is a multicomponent background-field theory supplemented, crucially, with a topological term. We also provide bulk sigma-model field theories of these phases and discuss a possible topological phase characterized by the thermal analog of the magnetoelectric effect.
\end{abstract}

DOI: 10.1103/PhysRevX.3.011016

\section{INTRODUCTION}

Following the discovery of topological insulators (TIs) [1], intense theoretical efforts have resulted in a good understanding of the topological phases of free fermions, including a complete classification of such phases that are stable to disorder [2]. In these phases, the bulk appears to local probes as a rather conventional gapped state. The surface is gapless, however, unless one of the symmetries protecting the phase is broken.

In contrast, our understanding of the topological phases of interacting particles is much less complete. The fractional quantum Hall effect has inspired much work on phases with topological order [3]. These phases have the remarkable property that the degeneracy of their ground states depends on the topology of the space on which they are defined. These phases are often associated with edge states, but, additionally, excitations with exotic statistics

Published by the American Physical Society under the terms of the Creative Commons Attribution 3.0 License. Further distribution of this work must maintain attribution to the author(s) and the published article's title, journal citation, and DOI. also occur in the bulk. Moreover, these phases of interacting particles are characterized by a topological entanglement entropy, implying long-range quantum entanglement in the ground state. It seems appropriate to exclude such phases from a minimal generalization of topological insulators to interacting systems.

We define a short-range entangled (SRE) state [4] as a gapped state with a unique ground state on all closed manifolds, i.e., one that has no topological order. In the presence of interactions, do new SRE phases appear that share the same symmetry but differ at the level of topology? A possible distinction, for example, is the presence of protected states at the boundaries.

In this paper, we study the physics of such SRE phases in systems of interacting bosons. For bosons, the noninteracting limit is a simple condensate so that interactions are necessary to stabilize gapped phases. Thus, we necessarily need to free ourselves from the crutch of free-fermion Hamiltonians and band topology on which most current discussions of topological insulators are based. Studying bosonic generalizations of topological insulators is potentially a useful step toward solving the harder problem of interaction-dominated fermionic topological insulators. 
Several examples of interacting-boson systems exist. A very natural realization of a strongly interacting boson system is a quantum magnet made up of localized quantum spins on a lattice. In that context, the phases we are interested in may be dubbed "topological paramagnets." They have a bulk gap and no bulk topological order or fractional quantum numbers, but they have protected surface states, all features in close analogy to electronic topological insulators. The topological paramagnet should be distinguished from a more familiar (and more exotic) paramagnet- the quantum spin-liquid state-which has been extensively discussed in the literature. Quantum spin-liquid states have either bulk topological order or gapless excitations. Other realizations of strongly interacting bosons are, of course. provided by ultracold atoms in optical lattices.

A famous example of a topological paramagnet already exists: the spin-1 Haldane (or AKLT) chain, which has a bulk gap and no fractionalization but has dangling spin-1/2 moments at the edge that are protected by symmetry [6]. Using a powerful matrix-product representation of gapped states [7-10], topological phases in one dimension are completely classified by the second group cohomology of symmetry group $\mathrm{G}$. In $2 \mathrm{D}$ and $3 \mathrm{D}$, such rigorous results are not available. However, Kitaev pointed out that 2D SRE phases of bosons with chiral edge states are nevertheless possible [11]. Recently, Chen et al. [5] have proposed that SRE topological phases of bosons protected by symmetry are captured by the higher-dimensional cohomology groups. While this insight provides an efficient mathematical scheme to enumerate phases, unfortunately, their properties are not transparently obtained.

Progress on clarifying the physics of these states has subsequently been made by several authors. Levin and $\mathrm{Gu}$ [12] explicitly studied a specific example in 2D protected by $Z_{2}$ symmetry. $2 \mathrm{D}$ SRE topological phases were described using a simpler Chern-Simons approach [13], which provided a field theory and explicit edge theories of these states. It was found that bosons with a conserved global U(1) but no other symmetries can display an "integer quantum Hall state" with quantized Hall conductance predicted to be even-integer multiples of $q^{2} / h$, i.e., $\sigma_{x y}=2 n q^{2} / h$, where $q$ is the elementary charge of the bosons [13]. A simple physical realization of such a phase of two-component bosons in the lowest Landau level has recently been provided [14]. Other physical interpretations have also been described [15,16]. Thus, some defining properties of several symmetry-protected topological (SPT) phases in 1D and 2D are now understood. However in 3D, although the cohomology classification predicts various SPT phases, including bosonic generalizations of topological insulators, the physical properties of SPT phases have not thus far been elucidated. This elucidation is our primary task in this paper. Potentially, our approach could also be used to classify 3D bosonic SPT phases, which we leave to future work. We note, however, that, for all symmetry classes that we considered both with time-reversal $\left(Z_{2}^{T}, \mathrm{U}(1) \times Z_{2}^{T}, \mathrm{U}(1) \rtimes Z_{2}^{T}\right)$ and without $\left(\mathrm{U}(1) \rtimes Z_{2}\right)$, we identify candidate phases that exhaust those predicted by the cohomology classification [5]. Further, we identify a possible topological phase that appears to be outside the classification of Ref. [5].

Much of our discussion focuses on a theory of the novel surface states of these 3D bosonic topological insulators. We construct effective Landau-Ginzburg field theories of these surface states. We also identify bulk field theories that correctly yield the proposed surface theory. A key feature of this surface theory is that it does not admit a trivial gapped symmetry-preserving surface phase. The surface either spontaneously breaks symmetry, or, if gapped, develops surface topological order (even though there is no bulk topological order). Other, more exotic symmetrypreserving states with gapless excitations are also possible. In all these cases, the defining global symmetries are implemented in a way not allowed in strictly $2 \mathrm{D}$ systems. As a specific example, consider insulating bosonic phases with the symmetries of a topological insulator: charge conservation and time-reversal symmetry [formally denoted as $\left.\mathrm{U}(1) \rtimes Z_{2}^{T}\right]$. For these phases, the surface can break a symmetry, e.g., by forming a surface superfluid. Alternatively, the surface can remain insulating, while breaking time-reversal symmetry. The bulk is assumed to retain the symmetry. Then these surface-ordered phases can reflect their special origin by exhibiting features that are forbidden in purely $2 \mathrm{D}$ phases. For example, we show that the vortices of the surface superfluid mentioned above are, in a precise sense, fermionic. Furthermore, the surface insulator with broken time-reversal symmetry is shown to have a Hall conductance of $\sigma_{x y}= \pm 1$ (in units of $q^{2} / h$ ), in contrast to the 2D integer quantum Hall phases of bosons, which are allowed only to even-integer Hall conductance.

Equivalently, the surface quantum Hall effect may be considered a quantized 3D response: the magnetoelectric polarizability $\theta$. Recall, in the context of free-fermion topological insulators with broken time-reversal on the surface that gaps the surface states, that a quantized magnetoelectric effect appears [17] that is captured by the topological theta term:

$$
\mathcal{L}_{\theta}=\frac{\theta}{4 \pi^{2}} \vec{E} \cdot \vec{B},
$$

where $h=c=e=1$ and $\vec{E}$ and $\vec{B}$ are applied electric and magnetic fields. For free-fermion topological insulators, $\theta=\pi(\bmod 2 \pi)$, corresponding to a half-integer Hall effect on the surface. The $2 \pi$ ambiguity in $\theta$ corresponds to the fact that one may deposit a fermionic integer quantum Hall layer on the surface [18].

Here, for bosonic topological insulators, $\theta$ is only defined modulo $4 \pi$, and the topological phase corresponds to $\theta=2 \pi$. This theta value implies, for example, that the domain wall between opposite time-reversal-symmetrybreaking regions on the surface induces a protected 
mode, which is the edge state corresponding to the $\sigma_{x y}=2$ quantized Hall effect of bosons. The $4 \pi$ ambiguity in $\theta$ corresponds to the fact that one may deposit an integer quantum Hall layer of bosons on the surface, which must have an even-integer Hall conductance.

A symmetry-preserving surface state can be accessed from the superfluid by condensing vortices that transform trivially under the symmetry. However, as the vortex of the surface superfluid has fermionic statistics (in the sense discussed in Sec. IV D, it cannot condense. This unusual nature of the vortices precludes the possibility of a trivial gapped surface insulator. The fermionic vortices can, of course, pair and condense. However, as is well known [19], paired-vortex condensation leads to a 2D state with topological order (described in the present context by a deconfined $Z_{2}$ gauge theory). We show that this surface topological order realizes symmetry in a manner not allowed in strictly 2D systems.

Exotic gapless surface states that preserve all symmetries are also conceivable. For example, the gapless surface state may intuitively be viewed as a quantum Hall state that fluctuates between $\sigma_{x y}= \pm 1$. The theory of such a state is constructed using a network model that captures the quantum-phase transition between distinct integer quantum Hall states of bosons. The same approach, when applied to fermionic topological insulators, correctly yields the single-Dirac-cone surface state. The field theory thus obtained of the bosonic model poised at the transition naturally leads to the required surface theory, which is closely related to the deconfined-quantum-critical theory [20] previously proposed in the context of frustrated quantum magnets.

$3 D$ field theory.-The general arguments above are shown to be consistent with the following $d=3$ field theory:

$$
\begin{aligned}
2 \pi \mathcal{L}_{3 D}= & \sum_{I} \epsilon^{\mu \nu \lambda \sigma} B_{\mu \nu}^{I} \partial_{\lambda} a_{\sigma}^{I} \\
& +\Theta \sum_{I, J} \frac{K_{I J}}{4 \pi} \epsilon^{\mu \nu \lambda \sigma} \partial_{\mu} a_{\nu}^{I} \partial_{\lambda} a_{\sigma}^{J},
\end{aligned}
$$

where the index $I$ refers to boson species; bosons' four currents are represented by $j^{\mu I}=\frac{1}{2 \pi} \epsilon^{\mu \nu \lambda \sigma} \partial_{\nu} B_{\lambda \sigma}^{I}$; and the curl of $a^{I}$ represents the vortex lines. The first backgroundfield (BF) term [21] just represents the $2 \pi$ phase factor of taking a particle around its vortex. The key topological properties, however, are determined by the second $\mathrm{BF}$ term, which attaches quantum numbers to vortices. To avoid topological order at the surface and to ensure bosonic excitations, $\operatorname{det} K=1$ and diagonal entries are even integers. In most cases, we take two species with $K=\sigma_{x}$. Here, $\Theta \rightarrow$ $\Theta+2 \pi$ is assumed to lead to an equivalent theory that differs only in details of surface termination. Furthermore, time-reversal symmetry constrains $\Theta=0, \pi$, the latter being the topological phase. Note that $\Theta$ for the internal gauge fields $a^{I}$ is distinguished from $\theta$ for the external electromagnetic field discussed above. Coupling to an external electromagnetic field allows one to obtain the quantized magnetoelectric effect discussed above. Related theories have appeared in the context of 3D topologically ordered phases and superconductors [22] where only the first BF term in Eq. (2) appears with a different coefficient. On the other hand, the field theory discussed in Ref. [23] retains only the second term, which leads to gapless excitations in the bulk, as noted in Ref. [24], which in turn differ from the gapped phases of interest here. Thus, it is important to combine both the first and the second terms.

Recently, it was proposed that free fermion topological insulators are captured by similar theories [25], with a single component field and the first term of Eq. (2) along with coupling to the external field. The surface states in such theories were argued to be bosonized Dirac fermions $[25,26]$. While this is an intriguing idea, we point out (see Appendix A) certain problems with the identification of a metallic surface in Ref. [25]. Moreover, identification of a bulk fermionic operator is also problematic in this theory $[25,26]$. One approach, taken in a recent paper [27], is to regard this as a partial theory that provides a purely hydrodynamic description that excludes fermionic excitations altogether. Thus, finding a complete, effective field-theory description for 3D fermionic topological insulators remains an open problem.

We also display continuum field-theoretic models in $D=3+1$ dimension that realize some of the topological phases we describe. These models are obtained as perturbations of nonlinear sigma models in the presence of a topological theta term. The theta term has the effect of endowing topological-defect configurations of the continuum fields with nontrivial global quantum numbers. We show the connection to the topological BF theory and to the theory of protected surface states.

Possible phases with half-quantized surface thermal Hall effect.-Interestingly, our general approach and the theory in Eq. (2) both predict a new 3D SPT phase, protected by time-reversal symmetry, that is not found within the cohomology classification of Chen et al. [5]. Just as the quantized Hall effect of $d=2$ SRE bosons immediately constrained the physics in 3D, an analogous argument for thermal Hall conductance can be made. In $d=2$, this is quantized to 8 times the quantum of thermal conductance: $\kappa_{x y} / T=8 n \frac{\pi^{2} k_{B}^{2}}{3 h}$ for a 2D SRE phase of bosons. A realization of $n=1$ is the Kitaev $E_{8}$ state [11], with 8 chiral bosons at the edge. Therefore, a 3D phase protected by timereversal symmetry can be conceived of, on the surface of which a domain wall between opposite $T$ breaking regions hosts 8 chiral boson modes and which is described by Eq. (2), with the 8D $K^{E_{8}}$ matrix discussed in Ref. [13]. This putative phase lies outside the cohomology classification of Chen et al. [5], which reports a single nontrivial topological phase with this symmetry, for which a different candidate, with $K=\sigma_{x}$, is identified in Sec. VII. 


\section{OVERVIEW}

As this paper is long and discusses bosonic SPT phases from several points of view, it is helpful to provide an overview. We seek an effective Landau-Ginzburg description of the surface of a 3D SPT phase. We employ various approaches that, satisfyingly, lead to consistent conclusions. In this section, we motivate these different approaches as preparation for the rest of the paper.

A key physical requirement of the surface theory is that there be no trivial gapped-symmetry-preserving surface phase. This feature is reminiscent of the Lieb-SchultzMattis (LSM) theorem [6] and its generalization [28,29] to states of bosonic systems at a fractional filling on clean 2D lattices. Indeed, in both cases, either a symmetry must be broken or there is topological order, or there is more exotic long-range entanglement, possibly with protected gapless excitations. There is one important difference, however. For the surface states discussed in this paper, the trivial insulating phase does not exist even in the presence of disorder that breaks translation symmetry. They are protected by internal symmetries rather than by lattice-translation symmetry (as is the case for the LSM theorem). Nevertheless, we exploit insights from existing effective field theories of clean lattice bosons that build in the LSM restrictions in order to construct the desired surface theory of the bosonic topological insulator.

We begin with a physical discussion of the constraints on quantized electrical and thermal responses in 3D SRE bosonic insulators, imposed by our knowledge of 2D SRE phases (Sec. III). In particular, we argue that the quantized magnetoelectric coupling for bosonic topological insulators $(\theta=2 \pi)$ is double that of the fermionic case $(\theta=\pi)$, which parallels the doubling of Hall conductance $[13,14,16]$ for $2 \mathrm{D}$ integer states of bosons compared to fermions.

Next, we borrow an approach that is useful in classifying SPT phases of 2D bosons. There, the edge states are typically described by a 1D Luttinger liquid theory. What makes them special is that symmetry acts on these edge states in a way that is impossible to realize in a purely $1 \mathrm{D}$ system [13-16]. This property ensures that all perturbations that lead to a gapped phase also break symmetry. By analogy, to discuss a 3D SPT phase, we model the 2D surface by a conventional 2D theory of bosons (e.g., an XY model). However, we identify symmetry operations that are forbidden in a conventional 2D system by demanding that the 2D system can never enter a trivial gapped phase.

As a useful device, consider an enlarged $\mathrm{U}(1) \times \mathrm{U}(1)$ global symmetry, along with time-reversal symmetry $\mathcal{T}$ (technically, $[\mathrm{U}(1) \times \mathrm{U}(1)] \rtimes Z_{2}^{T}$ ), and eventually break it down to the symmetry of interest. Physically, this enlarged symmetry corresponds to separate conservation of two species of bosons: species 1 and 2. Consider the surface of a 3D SPT phase with a broken symmetry, e.g., a condensate of species-1 bosons that breaks the first U(1) symmetry. This superfluid must also be unusual in that it cannot be connected to a fully symmetric insulator. Guidance from effective field theories of clean 2D lattice bosons at fractional filling suggests thinking in terms of the vortices of the superfluid. Since the insulating state is obtained by condensing vortices, we can ask, "What vortex properties provide the required obstruction?" Unlike a particle, a vortex is a nonlocal object and can transform projectively under the remaining $\mathrm{U}(1)$ and $\mathcal{T}$ symmetry. Vortices with projectively realized symmetries provide an obstruction to realizing a trivial insulator and can describe the surface of an SPT phase. In this example, the projective transformation requires that the two species of vortices $\left(\psi_{ \pm}\right)$carry halfcharge of species 2 , and these two species are exchanged by time-reversal symmetry. A minimal theory of these vortices is obtained by representing the density of the condensed species 1 by the curl of a vector potential $N_{1}=\left(\partial_{x} \alpha_{y}-\partial_{y} \alpha_{x}\right) / 2 \pi$, and this vector potential couples minimally to the vortices:

$$
\begin{aligned}
\mathcal{L}_{\text {edge } 2 \mathrm{D}}= & \sum_{\sigma= \pm}\left|\left(\partial_{\mu}-i \alpha_{\mu}-\frac{i \sigma}{2} A_{2 \mu}\right) \psi_{\sigma}\right|^{2} \\
& +\frac{\epsilon^{\mu \nu \lambda}}{2 \pi} A_{1 \mu} \partial_{\nu} \alpha_{\lambda}+K\left(\partial_{\mu} \alpha_{\nu}-\partial_{\nu} \alpha_{\mu}\right)^{2},
\end{aligned}
$$

where we have inserted external electromagnetic fields $A_{1}$, $A_{2}$ that couple to the two conserved currents. To obtain an insulating surface, we must condense the vortices, but this action inevitably breaks symmetry. Since the vortices transform into one another under time reversal, one cannot condense one species and not the other. Condensing them both, however, implies breaking the other U(1) symmetry, since

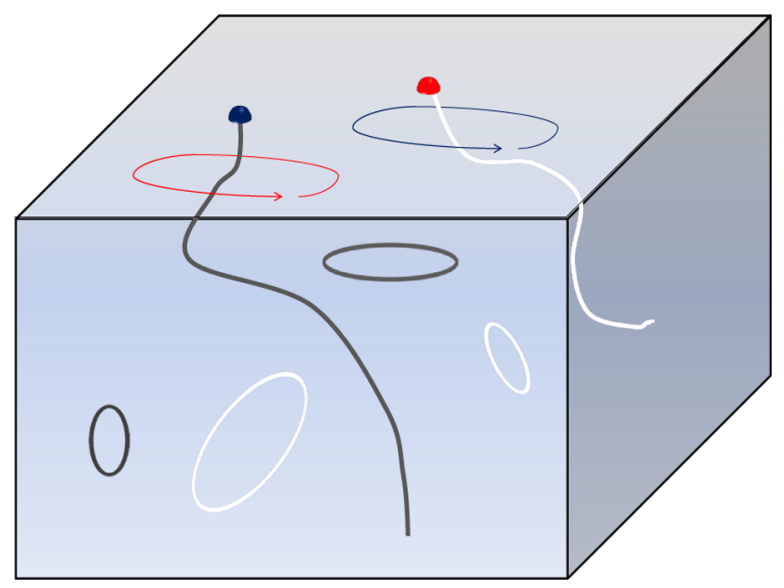

FIG. 1. Schematic depiction of a 3D symmetry-protected topological phase, with two conserved species of bosons $\left[\mathrm{U}(1) \times \mathrm{U}(1)\right.$, blue and red] and time-reversal symmetry $\left(Z_{2}^{T}\right)$. The bulk is insulating and corresponds to a condensate of vortices of both species (shown as black and white loops). In the topological phase, the vortex line of one species that ends on a surface carries half charge of the other species. Such a surface, it may be argued, does not have a trivial gapped phase, where the symmetries are preserved. Pictorially, the vortex lines may be viewed in 1D Haldane phases, with half-charged end states. 
the vortices carry the charge of species 2 . A third option is to condense pairs of vortices, but this results in $Z_{2}$ topological order [19]. This discussion points to a schematic picture of the SPT phase as shown in Fig. 1. A bulk insulator may be regarded as a condensate of vortex loops. However, the special feature here is that the condensed vortex lines carry a half charge at their ends when they intersect the surface. The vortex lines, viewed as 1D objects, are in a topological state analogous to the Haldane phase, and thus carry edge states.

Note that, if a single bosonic vortex species was present at low energies, we could perform a duality back to the usual boson phase variables. However, having multiple vortex fields demands a dual description like the one above. In fact, an identical theory appeared in the discussion of deconfined quantum critical points [20,30,31]; however, there the symmetries included both internal and spatial symmetries. Here all symmetries are internal and hence are restricted to the surface of a 3D topological phase.

Let us consider in more detail the insulating surface obtained on breaking time-reversal symmetry. This breaking is obtained by condensing just one species of vortex, say, $\psi_{-}$, in Eq. (3), which forces $\alpha=\frac{1}{2} A_{2}$. When substituted into the action above, this calculation yields the electromagnetic response

$$
\mathcal{L}_{\mathrm{em}}=\frac{1}{4 \pi} A_{2 \mu} \epsilon_{\mu \nu \lambda} \partial_{\nu} A_{1 \lambda}
$$

If the separate species are now identified with a single conserved charge, we can set $A_{1}=A_{2}=A$, which yields $\sigma_{x y}=1$ on the surface, indicating a magnetoelectric response $\theta=2 \pi$, as predicted. In the absence of timereversal breaking, the surface may be assumed to fluctuate between $\sigma_{x y}= \pm 1$. We provide an alternate derivation of the same surface theory [Eq. (3)] by modeling it using a network model, poised at the transition point between two bosonic integer quantum Hall states. This model is analogous to obtaining the surface state of the $3 \mathrm{D}$ fermionic topological insulators, the single Dirac cone, by a network model at the critical point between integer quantum Hall plateaus [32] in a clean system. In both cases, the timereversal symmetry automatically tunes the system to criticality. For the bosonic SPT surface, the resulting theory is an $\mathrm{O}(4)$ nonlinear sigma model with a topological term, as in the Euclidean Lagrangian

$$
\begin{aligned}
\mathcal{L}_{\text {edge } 2 \mathrm{D}}^{\prime}= & \frac{1}{2 \kappa} \operatorname{Tr}\left(\partial_{\mu} g^{\dagger} \partial_{\mu} g\right) \\
& +i \frac{\pi}{24 \pi^{2}} \epsilon^{\mu \nu \lambda} \operatorname{Tr}\left[\left(g^{\dagger} \partial_{\mu} g\right)\left(g^{\dagger} \partial_{\nu} g\right)\left(g^{\dagger} \partial_{\lambda} g\right)\right]
\end{aligned}
$$

where the $\mathrm{O}(4)$ vector has been written in terms of an $\mathrm{SU}(2)$ matrix $g$. Reassuringly, this model has been argued in Ref. [33] to be equivalent to Eq. (3) once appropriate anisotropies are introduced. These two descriptions of surface properties of SPT phases are discussed in Sec. IV along with the connection between them.

We also emphasize the properties of a gapped symmetric surface state obtained by condensing paired vortices. This state has surface topological order described by a deconfined $Z_{2}$ gauge theory (even though the bulk has no such order). This state provides a particularly simple perspective on why a trivial gapped symmetric phase is forbidden at the surface. Indeed, we show that bosonic topological quasiparticles of this state carry fractional quantum numbers. Destroying the topological order by condensing one of these quasiparticles necessarily breaks a symmetry. Not surprisingly, we show that the implementation of symmetry in this surface topological ordered state is distinct from what is allowed in strict 2D systems.

In Sec. V, we discuss bulk theories that are consistent with the surface descriptions above. We describe SPT phases in quantum magnets-the ones we dubbed topological paramagnets-protected by other symmetries such as time-reversal $\left(Z_{2}^{T}\right)$, or time-reversal along with one component of spin rotation $\left[\mathrm{U}(1) \times Z_{2}^{T}\right]$ in Sec. VI. (Appendix $\mathrm{C}$ contains an example without time-reversal symmetry $\left[\mathrm{U}(1) \rtimes Z_{2}\right]$. Section VII closes the paper with the discussion of a new topological phase that is predicted by this approach, a 3D extension of Kitaev's $E_{8}$ state, along with some additional comments.

\section{TRANSPORT PROPERTIES OF 3D BOSONIC TOPOLOGICAL INSULATORS: GENERAL CONSTRAINTS}

We begin by considering a system of interacting bosons in $d=3$ space dimensions in the presence of time-reversal and particle-number-conservation symmetries. Specifically, let us consider the situation in which the boson field $b$ carries charge 1 under a global U(1) symmetry and transforms as $b \rightarrow b$ under time reversal. The corresponding symmetry group is $\mathrm{U}(1) \rtimes Z_{2}^{T}$. Assume the system is in a gapped insulating phase (at least in the absence of any boundaries) and that there is a unique ground state on topologically nontrivial manifolds. For any such insulator in 3D, the effective Lagrangian for an external electromagnetic (EM) field obtained by integrating out all the matter fields takes the form

$$
\mathcal{L}_{\text {eff }}=\mathcal{L}_{\text {Max }}+\mathcal{L}_{\theta} .
$$

The first term is the usual Maxwell term and the second is the "theta" term in Eq. (1).

Several properties of the theta term are well known. First, under time reversal, $\theta \rightarrow-\theta$. Next, on closed manifolds, the integral of $\frac{1}{4 \pi^{2}} \vec{E} \cdot \vec{B}$ is quantized to be an integer so that the quantum theory is periodic under $\theta \rightarrow \theta+2 \pi$. These two facts together imply that time-reversalsymmetric insulators have $\theta=n \pi$, with $n$ an integer. Trivial time-reversal-symmetric insulators have $\theta=0$, while free-fermion topological insulators have $\theta=\pi$. 
If we allow for a boundary to the vacuum and further assume that the boundary is gapped (if necessary by breaking time-reversal symmetry), then the $\theta$ term leads to a surface Hall conductivity of $\frac{\theta}{2 \pi}$. To see this, assume a boundary (at, say, $z=0$ ), then $\theta=\theta(z)$ is zero for $z<0$ and constant $\theta$ for $z>0$. The action associated with the $\theta$ term is

$$
\begin{aligned}
S_{\theta} & =\frac{1}{8 \pi^{2}} \int d^{3} x d t \theta(z) \partial_{\mu} K^{\mu} \\
& =-\frac{1}{8 \pi^{2}} \int d^{3} x d t \frac{d \theta}{d z} K^{z} \\
& =\frac{\theta}{8 \pi^{2}} \int_{\partial B} d^{2} x d t \epsilon^{z \nu \lambda \kappa} A_{\nu} \partial_{\lambda} A_{\kappa},
\end{aligned}
$$

where $A$ is the external electromagnetic potential and $K^{\mu}=\epsilon^{\mu \nu \lambda \kappa} A_{\nu} \partial_{\lambda} A_{\kappa}$. Equation (9) is a surface ChernSimons term and leads to a Hall conductivity of $\theta / 2 \pi$.

For fermion topological insulators, $\theta=\pi$ so that the surface $\sigma_{x y}=\frac{1}{2}$. If we shift $\theta \rightarrow \pi+2 n \pi$, then the surface $\sigma_{x y}=\left(n+\frac{1}{2}\right)$. This shift corresponds to simply depositing an ordinary integer quantum Hall state of fermions at the surface of this insulator. Hence, this shift should not be regarded as a distinct bulk state. The only nontrivial possibility is $\theta=\pi$.

Now let us consider bosonic insulators. Again, $T$ reversal and periodicity imply that $\theta=n \pi$ and that a surface $\sigma_{x y}=n / 2$. A crucial observation is that now $\theta=2 \pi$ must be regarded as distinct from $\theta=0$. At $\theta=2 \pi$, the surface $\sigma_{x y}=1$. However, this Hall conductivity cannot be obtained from the surface of the $\theta=0$ insulator by depositing any $2 \mathrm{D}$ integer quantum Hall effect (IQHE) state of bosons. Recent work $[13,14]$ has shown that 2D IQHE states of bosons necessarily have $\sigma_{x y}$ even. (See Ref. [14] for a simple argument.) Thus, the surface state of the $\theta=2 \pi$ boson insulator is not a trivial 2D state but rather requires the presence of the $3 \mathrm{D}$ bulk.

Therefore, $\theta=2 \pi$ necessarily corresponds to a nontrivial 3D bosonic TI. $\theta=4 \pi$ is trivial, however, as then the surface state can be regarded as a $2 \mathrm{D}$ bosonic IQHE state. One may still obtain a 3D topological phase, but the topology is not manifest in the electromagnetic response.

We can sharpen and generalize this result. Under $T$ reversal, as $\theta \rightarrow-\theta, n \pi \rightarrow-n \pi$. As the bulk state is $T$ reversal invariant, we require that the surface state at $\theta=-n \pi$ be obtainable from the surface state at $\theta=+n \pi$ by depositing a $2 \mathrm{D} \mathrm{IQHE}$ boson state. Let us characterize the surface state by both its electrical and its thermal Hall conductivities $\left(\sigma_{x y}, \kappa_{x y}\right)$. Under $T$ reversal, both Hall conductivities change sign. The requirement described above then means that $\left(2 \sigma_{x y}, 2 \kappa_{x y}\right)$ must correspond to the allowed electrical and thermal Hall conductivities of a $2 \mathrm{D}$ boson IQHE state.
For $\theta=2 \pi$, it follows that $\sigma_{x y}=1, \kappa_{x y}=0$. It is thus "half" of the elementary 2D boson IQHE state.

For $\theta=\pi, 2 \sigma_{x y}=1$, which is not allowed for the 2D bosonic IQHE. It follows, therefore, that 3D bosonic TIs that have no "intrinsic topological order" cannot have $\theta=\pi$. It is, of course, very easy to construct such states [34-36] (or other states with fractional $\theta$ ) if we allow for fractionalization of the boson, but that violates our original assumption.

A 2D IQHE state with $2 \sigma_{x y}=8,2 \kappa_{x y}=8$ is allowed; it is discussed by Kitaev [11]. Thus, a 3D boson TI with surface $\sigma_{x y}=4, \kappa_{x y}=4$ is allowed. Combining these two types of fundamental states generates the allowed thermal and electrical Hall responses on the surface.

Later in this paper, we discuss how these results fit with the formal classification of SPT and other short-ranged entangled phases in 3D. For now, we reiterate the crucial observation of this section: A state with EM response of $\theta=2 \pi$ necessarily describes a topological insulator of $T$-reversal-symmetric bosons while $\theta=\pi$ requires the presence of "intrinsic topological order." In the next section, we study the properties of this $\theta=2 \pi$ boson topological insulator in detail.

\section{SURFACE THEORY OF 3D BOSONIC SPT PHASES}

In this section, we derive the nontrivial surface theory of one example of a 3D bosonic SPT phase. We soon specialize to the symmetries of the topological insulator: charge conservation and time-reversal symmetry $\left[\mathrm{U}(1) \rtimes Z_{2}^{T}\right]$ and exhibit a nontrivial topological phase in three dimensions, built purely of bosons. To begin, we assume that there are two species of bosons whose numbers are separately conserved and that there is enlarged $[\mathrm{U}(1) \times \mathrm{U}(1)] \rtimes Z_{2}^{T}$. Later, we break this enlarged symmetry to just $\mathrm{U}(1) \rtimes Z_{2}^{T}$ symmetry by including interspecies boson mixing terms in the Hamiltonian. A similar construction [13] has proven to be very powerful in $d=2$. We consider two approaches.

Projective vortices. - We exploit the fact that a bosonic SPT phase in $d$ dimensions has surface states that correspond to a conventional theory of bosons in $d-1$ dimensions except in the way that symmetries are implemented. For example, the edges of SPT phases of bosons in $D=2+1$ dimensions correspond to conventional 1D Luttinger liquids except for their unusual symmetry transformations [13-15]. We therefore consider a 2D bosonic state to model the surface and assume that the surface is a superfluid breaking one of the U(1) symmetries. Then, vortices of this condensate may transform under a projective representation of the remaining symmetry group. In a projective representation, even the identity element of the symmetry group induces a phase rotation. Hence, local operators, which can be physically measured, must remain unchanged under the identity operation of the symmetry group, since this operation corresponds to "doing 
nothing". However, vortices, which are nonlocal objects, can transform projectively.

One may attempt to restore the U(1) symmetry by condensing vortices. However, the projective transformation ensures that, when vortices condense, they necessarily break another symmetry. In this way, both the boson and vortex condensates lead to symmetry breaking, in line with our general expectation for the surface of a 3D SPT phase. It is important that vortices transform projectively so that they cannot be screened by bosons to obtain a trivial representation of the symmetry group. The projective representation is a generalization of the idea of quantumnumber fractionalization. For example, a particle with half charge changes sign under the $2 \pi$-phase rotation of bosons, implying a projective representation. Clearly, a half charge cannot be screened by any finite number of bosons. Projective representations were also recently used to classify SPT phases in $D=1+1$, where they correspond to the ends of gapped 1D topological phases. For example, the half-integer spin edge states of spin-1 Haldane chains furnish a projective representation of the rotation group. The reasoning above suggests a physical picture of a 3D SPT phase in which the vortex line in the bulk is similar to a Haldane-chain-type gapped phase, which requires low-energy states on the surface where the vortex ends. In this section, we specialize to the symmetries of the topological insulator. Then this procedure explicitly produces a topological phase characterized by the quantized magnetoelectric effect $\theta=2 \pi$.

Network model.-We directly implement the property discussed in Sec. III that, if the surface breaks $T$ reversal and is gapped, then it has quantized Hall transport. If $T$ reversal is not broken, a powerful approach to obtaining the surface theory is to start with the theory of the quantum phase-transition point between the two bosonic quantum Hall phases that correspond to the two $T$-broken surfaces. In the case of free-fermion topological insulators, a similar reasoning leads to the single Dirac-cone-surface state that describes the transition between the $\sigma_{x y}= \pm \frac{1}{2}$ states on the surface. For free fermions, the transition between these integer quantum Hall states is described by a ChalkerCoddington network model [37]. For the bosonic problem of interest here, we construct an analogous network model and show that it leads to a sigma model with a topological term.

The results of these two approaches are readily seen to be connected. In both cases, the field theories we obtain for the surface have been discussed previously in the context of deconfined quantum criticality. We here discuss the phase diagram of the surface states described by these field theories. When interspecies tunneling is included, the vortices of the two species of bosons are confined to each other. The resulting single vortex no longer transforms projectively under the physical symmetries. However, we argue that it is most conveniently viewed as a fermion. This unusual nature of the vortices precludes the possibility of obtaining a trivial insulating phase at the surface by condensing vortices.

\section{A. Surface states and projective vortices}

Consider a boson field at the surface with phase degree of freedom $\phi_{1}, b_{1}^{\dagger}=e^{i \phi_{1}}$. We assume that the bulk is insulating and that the surface is in the $x-y$ plane.

The surface theory could spontaneously break a global $\mathrm{U}(1)$ symmetry of boson number conservation (a surface superfluid) or it could stay insulating. More precisely, as the bulk is always assumed to be insulating, the vortex line loops have proliferated in the bulk. These vortex lines penetrate the surface at points that may be viewed as point vortices of the $2 \mathrm{D}$ surface theory since there is no vortex line tension in the insulating bulk. These point vortices are gapped when the surface is a superfluid. If, instead, they are condensed, the surface is insulating. To describe vortices, we move to a dual description $[38,39]$ in which we write the density and currents of the boson $b_{1}$ on the surface in terms of the field strengths of a gauge field $j_{1 \mu}=\epsilon^{\mu \nu \lambda} \partial_{\nu} \alpha_{2 \lambda} / 2 \pi$. In particular, the density of bosons is $n_{1}=\left(\partial_{x} \alpha_{2 y}-\partial_{y} \alpha_{2 x}\right) / 2 \pi$. (The reason for the subscript 2 on $\alpha$ will soon be apparent.) The boson insertion operators $e^{i m \phi_{1}}$ correspond to monopole insertion operators since they insert $2 \pi m$ magnetic flux. Now, the vortices $\Psi_{2}$ are particles that couple minimally to the gauge field $\alpha_{2}$. In general, multiple vortex species transform into each other under the symmetry operation. Let us label them by $i$, so $\left[\Psi_{2}\right]_{i}=\psi_{2 i}$. All these fields couple minimally to the gauge field.

Thus, we have for the dual surface theory

$$
\mathcal{L}_{\text {surf }}=\sum_{i, \mu}\left|\left(\partial_{1 \mu}-i \alpha_{2 \mu}\right) \psi_{2 i}\right|^{2}+V\left(\Psi_{2 i}\right)+\frac{1}{2 \kappa} f_{2 \mu \nu}^{2}+\ldots,
$$

where $f_{2 \mu \nu}=\left(\partial_{\mu} \alpha_{2 \nu}-\partial_{\nu} \alpha_{2 \mu}\right)$.

As argued above, one route to obtaining topological surface states is if the surface vortices transform under a projective representation of the remaining symmetry. Vortices that transform projectively under a global symmetry are actually quite familiar. They describe the generic situation of 2D bosons on a lattice, say, at some commensurate filling $[30,40]$. These projective vortices play a crucial role in the theory of deconfined quantum criticality.

We need consider only two component vortex fields $\Psi_{2}=\left(\psi_{2+}, \psi_{2-}\right)$ for the cases considered in this paper. The gauge-invariant combination $\Psi_{2}^{\dagger} \sigma^{+} \Psi_{2}=\psi_{2+}^{*} \psi_{2-}=$ $e^{i \phi_{2}}=b_{2}^{\dagger}$ then defines another bosonic field. Note that Eq. (10) closely resembles the action for a deconfined quantum critical point (noncompact $C P_{1}$ theory with easy-plane anisotropy) [20,41]. We demonstrate how Eq. (10) emerges as the theory for the surface states, and 
also, in the next section, describe a 3D bulk theory that leads to this edge theory.

\section{Surface states of a bosonic topological insulator: Symmetry $\mathrm{U}(1) \rtimes Z_{2}^{T}$}

The topological insulator has these symmetries: a conserved $\mathrm{U}(1)$ charge and $Z_{2}^{T}$ time-reversal symmetry. The semidirect product appears so that the charge-insertion operator $e^{i \phi}$ is invariant under time reversal, which involves both $\phi \rightarrow-\phi$ and $i \rightarrow-i$. Here, we construct the surface theory of a 3D topological phase that has these symmetries.

Let us begin with an enlarged symmetry, two species of bosons that are separately conserved. Consider a condensate of one species $b_{1}$. Vortices in this condensate are created by the field $\Psi_{2}$. We need to specify the projective representation for the vortices $\Psi_{2}$ and the transformation of the bosons $b_{1}^{\dagger}$. The remaining symmetry group $\mathrm{U}(1) \rtimes Z_{2}^{T}$ has a single projective representation (P1), which acts as follows: Under a U(1) rotation by angle $\epsilon$, the fields $\psi_{2 \pm} \rightarrow$ $e^{ \pm i \epsilon / 2} \psi_{2 \pm}$ and, under time-reversal $Z_{2}^{T}$, the fields $\psi_{2+} \rightarrow$ $\psi_{2-}^{*}$ and $\psi_{2-} \rightarrow \psi_{2+}^{*}$. Or, more compactly,

$$
\Psi_{2} \rightarrow e^{i(\epsilon / 2) \sigma_{z}} \Psi_{2}: \mathrm{U}(1), \quad \Psi_{2} \rightarrow \sigma_{x} \Psi_{2}^{*}: Z_{2}^{T} .
$$

Here, the $\sigma$ are the Pauli matrices in the standard representation. Thus, the vortices carry charge $\pm 1 / 2$ of bosons of the other species. The time-reversal symmetry that interchanges the two vortex fields ensures that the vortex charge is fixed exactly at half. It is impossible to "screen" this charge with regular integer charged bosons.

These transformation laws, of course, determine how the boson operator $b_{2}^{\dagger}=e^{i \phi_{2}}$ and how their density $n_{2}$ transforms. We also need to specify how the bosons $b_{1}^{\dagger}=e^{i \phi_{1}}$ and how density $n_{1}$ transforms. The symmetry transformations are

$$
\phi_{1,2} \rightarrow \phi_{1,2}+\epsilon \text { for U(1), } \phi_{1,2} \rightarrow-\phi_{1,2} \text { for } Z_{2}^{T} .
$$

The conjugate boson numbers therefore transform as

$$
n_{1,2} \rightarrow n_{1,2} \text { for } \mathrm{U}(1), \quad n_{1,2} \rightarrow n_{1,2} \text { for } Z_{2}^{T} .
$$

A necessary compatibility check is that Eq. (11) is invariant under the symmetry operation, which can be verified for these transformations. For example, timereversal symmetry is implemented via Eq. (10) on the vortex fields, which is compatible with $n_{2}$ (and hence $\alpha_{2}$ ) remaining invariant while $i \rightarrow-i$ under time reversal. Moreover, since the bosons carry charge, the monopole insertion operators are forbidden.

Condensing single vortices then breaks symmetry, as described below. This situation is best analyzed by assuming separate number conservation of each species of boson, $n_{1}, n_{2}$, in which case they can be coupled to an external gauge potential $A_{1}, A_{2}$. Then the effective Lagrangian equation (10) reads

$$
\begin{aligned}
\mathcal{L}_{1}= & \sum_{s= \pm}\left|\left(\partial_{\mu}-i \alpha_{2 \mu}-i \frac{s A_{2}}{2}\right) \psi_{2 s}\right|^{2}+\ldots \\
& +\frac{1}{2 \kappa_{1}}\left(\epsilon_{\mu \nu \lambda} \partial_{\nu} \alpha_{2 \lambda}\right)^{2}+\frac{1}{2 \pi} A_{1 \mu} \epsilon_{\mu \nu \lambda} \partial_{\nu} \alpha_{2 \lambda} .
\end{aligned}
$$

The $\psi_{2 \pm}$ are vortex fields of $b_{1}$, which carry half charge of boson species 2 . The flux of the gauge field $\alpha_{1}$ is precisely the conserved density of species 1 , hence, the last term in the above action where the external probe gauge field $A_{1}$ couples to this current. This action needs to be modified by including all symmetry-allowed perturbations. We do this and analyze the possible phases in the rest of this section, but, as a preview, consider the effect of breaking timereversal symmetry by condensing just one species of vortex, say, $\psi_{2-}$. Anticipating a single charge, consider an external field that couples equally to the two charge densities $A_{1}=A_{2}=A$. The vortex condensate forces $\alpha_{2}=\frac{1}{2} A$, which, when substituted into the action above, yields the electromagnetic response $\frac{1}{4 \pi} A_{\mu} \epsilon_{\mu \nu \lambda} \partial_{\nu} A_{\lambda}$, which yields $\sigma_{x y}=1$ on the surface, indicating a magnetoelectric response $\theta=2 \pi$, as advertised. We now change track and obtain the same surface theory from a very different point of view.

\section{B. Construction of the network model}

The general considerations of Sec. III show that a timereversal-symmetric boson insulator with electromagnetic response characterized by $\theta=2 \pi$ is in a topologicalinsulator phase. This key result relies on the observation that, if $\mathcal{T}$ is broken at the surface to gap it out, then such a state has a quantized electrical Hall conductivity $\sigma_{x y}=$ \pm 1 , and a thermal Hall conductivity $\kappa_{x y}=0$. What if $T$ reversal is not explicitly broken at the surface? The surface can then potentially be gapless. What then is the nature of the resultant theory? To construct this theory, it is extremely instructive to learn from the example of the free-fermion $T$ reversal-symmetric topological insulator. In that case, if $T$ is explicitly broken to gap out the surface, then we obtain $\sigma_{x y}= \pm \frac{1}{2}$. When $T$ is unbroken, it is possible to get a single massless Dirac cone, which is exactly the low-energy theory of the transition between two integer quantum Hall plateaus of fermions in $d=2$. Generically, we can tune the chemical potential to move away from the Dirac point to get a Fermi surface that encloses the Dirac point.

Note that the quantized Hall conductance jumps by 1 across the integer quantum Hall plateau transition. When applied to the surface of the 3D fermionic topological insulator, the transition connects the two possible $T$-breaking surface states that go into each other under time reversal. Thus, each such surface state must be assigned $\sigma_{x y}= \pm \frac{1}{2}$ and the critical theory itself is timereversal invariant. In contrast, when applied to strictly $2 \mathrm{D}$ systems, the network model describes a transition between plateaus with $\sigma_{x y}=0$ and $\sigma_{x y}=1$. 
The familiar free-fermion example gives us a crucial clue for constructing the theory of the $T$-reversal symmetric surface state of the boson topological insulator. First, construct the low-energy theory of the $d=2$ integer quantum Hall state of bosons as a potential candidate for the gapless surface state of the 3D topological insulator. Then add perturbations allowed by symmetry to obtain the generic surface theory. Across the boson integer quantum Hall transition, $\sigma_{x y}$ jumps by 2 . As for the fermionic example, when this transition is realized at the surface of the bosonic topological insulator, the two plateau states on ether side of the transition are related by $T$ reversal and must be assigned $\sigma_{x y}= \pm 1$, consistent with our earlier arguments.

With this motivation, we now study the IQHE plateau transition of bosons in $d=2$.

IQHE quantum phase transition of bosons in $d=2$.We now study the phase transition using a "network" model construction. We start with the theory of the edge state and couple together opposite edges. Let us warm up with the familiar example of the IQHE transition of fermions from a state with $\sigma_{x y}=1$ to one with $\sigma_{x y}=0$. The model, defined by Fig. 2, is described by the Euclidean action $\mathcal{S}=\int d x d t \mathcal{L}$

$$
\mathcal{L}=\sum_{i} \bar{c}_{i}\left(\partial_{\tau}-i s_{i} \partial_{x}\right) c_{i}-\sum t_{i}\left(\bar{c}_{i+1} c_{i}+\bar{c}_{i} c_{i+1}\right)
$$

with

$$
\begin{gathered}
t_{i \text { even }}=t_{e}, \\
t_{i \text { odd }}=t_{o}, \\
s_{i}=-(-1)^{i} .
\end{gathered}
$$

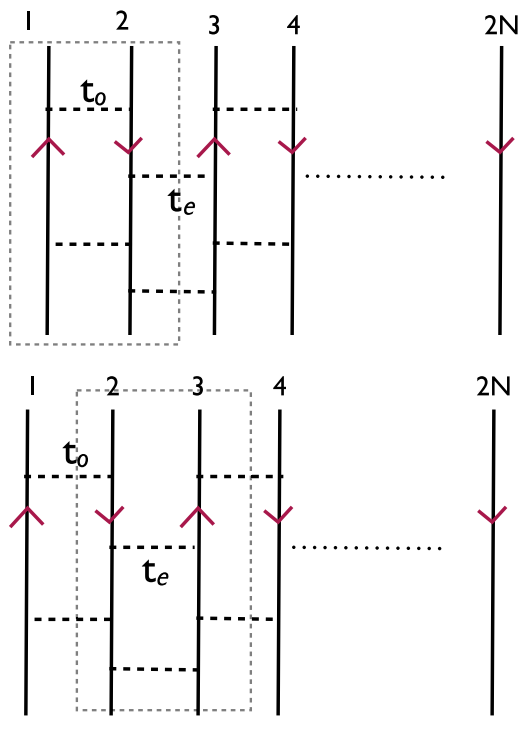

FIG. 2. Network model for the fermion IQHE transition. On the top, all chiral edge modes are paired to yield an ordinary insulator. On the bottom, there is an unpaired edge mode to yield an integer quantum Hall insulator.
The first term is the sum of the actions of a single chiral edge mode of the $\sigma_{x y}=1$ fermion IQHE state taken to propagate in opposite directions for adjacent $i$. The second term describes electron hopping between opposite-moving edge channels.

When $t_{e}<t_{o}$, all chiral edge channels are paired with partners and $\sigma_{x y}=0$. Conversely, if $t_{e}>t_{o}$, then all edge channels get paired except the two end ones and we get the fermion IQHE state with $\sigma_{x y}=1$. The transition occurs at $t_{e}=t_{o}$ and is readily seen to yield a single massless Dirac fermion if the continuum limit is taken in the $i$ direction.

Let us now repeat this construction for the bosonic IQHE transition. The edge theory for the boson IQHE state with $\sigma_{x y}=2, \kappa_{x y}=0$ has a pair of counterpropagating edge modes: One carries the charge and the other is neutral [13-15]. It is convenient to write the effective action of the edge as an $\mathrm{SU}(2)_{1}$ Wess-Zumino-Witten (WZW) theory:

$$
S_{\text {eff }}=\int d x d \tau \frac{1}{2 \lambda} \operatorname{tr}\left(\partial_{\mu} g^{\dagger} \partial_{\mu} g\right)+i S_{\mathrm{WZW}}[g] .
$$

Here, $g$ is a $2 \times 2$ matrix with entries

$$
g=\left(\begin{array}{cc}
b_{1} & -b_{2}^{*} \\
b_{2} & b_{1}^{*}
\end{array}\right)
$$

The $b_{1}, b_{2}$ are the two physical boson fields that form the IQHE quantum Hall state.

A network model capable of describing the boson IQHE state may now be written; it is defined by Fig. 3. Again, we have an array of opposite edge channels which are coupled together by boson hopping $-\sum_{a=1,2}\left(b_{i a}^{\dagger} b_{i+1, a}+\right.$ H.c. $) \propto$ $-\operatorname{tr}\left(g_{i}^{\dagger} g_{i+1}+\right.$ H.c $)$. The full effective action is then
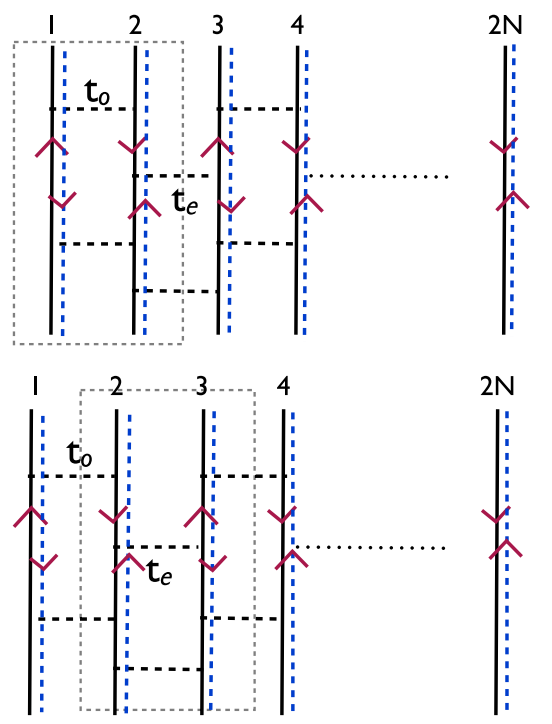

FIG. 3. Network model for the boson IQHE transition. Each edge channel now has a charged chiral mode and a counterpropagating neutral mode. The rest is the same as for fermions in Fig. 2. 


$$
\begin{gathered}
S=S_{0}+S_{W}+S_{t} \\
S_{0}=\int d x d \tau \frac{1}{2 \lambda} \sum_{i} \operatorname{tr}\left(\partial_{\mu} g_{i}^{\dagger} \partial_{\mu} g_{i}\right), \\
S_{W}=i \sum_{i} s_{i} S_{\mathrm{WZW}}\left[g_{i}\right] \\
S_{t}=-\sum_{i} t_{i} \operatorname{tr}\left(g_{i}^{\dagger} g_{i+1}+\text { H.c. }\right)
\end{gathered}
$$

with $s_{i}$ and $t_{i}$ as before. If $t_{o} \gg t_{e}$, we obtain the trivial insulator, while, if $t_{e} \gg t_{o}$, we get the boson IQHE state. The transition occurs at $t_{e}=t_{o}$. A low-energy theory of the transition is obtained by taking the continuum limit in the $i$ direction. As the opposite-moving edge channels have opposite WZW terms, they nearly cancel, and it is necessary to carefully sum them. Fortunately, precisely this sum was performed in Ref. [33] where the same model arose in a different context. The result is the effective $D=2+1$ dimensional action

$$
S_{\text {eff }}=\int d^{3} x \frac{1}{2 \kappa} \operatorname{tr}\left(\partial_{\mu} g^{\dagger} \partial_{\mu} g\right)+i \pi \mathcal{L}_{\theta}[g] .
$$

The second term is a $\theta$ term for the $\mathrm{SU}(2)$-matrix-valued field $g$ in $2+1$ dimensions corresponding to $\Pi_{3} \mathrm{SU}[(2)]=Z$. In the present context, our calculation has yielded this term at $\theta=\pi$.

We do not, of course, have full SU(2) symmetry rotating between $b_{1}$ and $b_{2}$ in the microscopic system. For the time being, let us assume that we have $\mathrm{U}(1) \times \mathrm{U}(1)$ symmetry corresponding to separate conservation of the $b_{1}, b_{2}$ bosons. Furthermore, let us also assume that there is a $Z_{2}$ symmetry interchanging $b_{1}$ and $b_{2}$. (Later, we relax all these assumptions.) Here, the results of Ref. [33] show that the field theory described above at $\theta=\pi$ maps onto the self-dual easy-plane noncompact $C P^{1}\left(\mathrm{NCCP}^{1}\right)$ model. Equivalently, it also maps onto a model of two species of spacetime loops with a phase $\pi$ associated with each linking of the two loop species.

The $\theta=\pi$ SU(2)-matrix field theory [with the $\mathrm{U}(1) \times \mathrm{U}(1)$ anisotropy] or the equivalent easy-plane $\mathrm{NCCP}^{1}$ model arises also in the theory of deconfined quantum criticality in two space dimensions. Remarkably, we see that the field theories describing the boson IQHE plateau transition (and hence the surface states of the 3D boson topological insulator) are closely related to the theory of deconfined quantum criticality. In the previous subsection, we obtained this connection from a different point of view. In the rest of the paper, we explore this connection in more detail and generality. For now, we merely point out that the results of Ref. [33] (see also Ref. [42]) show that the $\theta=\pi$ $\mathrm{SU}(2)$ matrix field theory in two space dimensions does not have a trivial gapped disordered phase. Its phases either break symmetry, are gapless, or have topological order. This phase structure is a hallmark of the surface state of a symmetry-protected topological phase-There is no trivial gapped phase that preserves all the symmetries.

Surface of the bosonic TIs: Field theories.-We now exploit our intuition about deconfined criticality to obtain the theory of possible surface states of the 3D boson TI, starting with the $\theta=\pi \mathrm{SU}(2)$-matrix field theory. We first describe a number of equivalent field-theoretic descriptions of the surface state, paying particular attention to the realization of the physical $\mathrm{U}(1) \rtimes Z_{2}^{T}$ symmetry. First, we note that the $\mathrm{SU}(2)$ matrix $g$ is related to the physical boson fields $b_{1,2}$ through

$$
g=\left(\begin{array}{cc}
b_{1} & -b_{2}^{*} \\
b_{2} & b_{1}^{*}
\end{array}\right) .
$$

Under the global U(1) symmetry, both bosons transform with charge 1 , i.e.,

$$
b_{1,2} \rightarrow b_{1,2} e^{i \varphi} .
$$

We implement time reversal by simply requiring that

$$
b_{1,2} \rightarrow b_{1,2} \text {. }
$$

In terms of the phases of the bosons, defined through $b_{1,2} \sim$ $e^{i \phi_{1,2}}$, and the conjugate bosons densities $n_{1,2}$, the symmetry transformations are the same as in Eqs. (12) and (13) so that we are indeed describing the same symmetry class in the two approaches. We remind the reader that the total number $n_{1}+n_{2}$ of the two boson species is conserved due to the global U(1) symmetry, but the relative number $n_{1}-n_{2}$ is, in general, not conserved. As promised, we first analyze the theory in a limit where this relative number is also conserved (so that there is $\mathrm{U}(1) \times \mathrm{U}(1)$ symmetry), and then we include interspecies tunneling terms to recover the generic case.

As argued in Ref. [33], the $\theta$ term of the SU(2) matrix field theory with $\mathrm{U}(1) \times \mathrm{U}(1)$ anisotropy has a simple interpretation: It is the phase that is picked up when the vortex of the boson $b_{1}$ is taken around the vortex of the boson $b_{2}$. At $\theta=\pi$, the two vortices are mutual semions. We may thus readily write a dual field theory in terms of the vortices $\Phi_{1 v, 2 v}$ of the two bosons $b_{1,2}$, respectively. This dual field theory has the structure

$$
\begin{gathered}
\mathcal{L}=\mathcal{L}_{1 v}+\mathcal{L}_{2 v}+\mathcal{L}_{\theta}+\mathcal{L}_{A}, \\
\mathcal{L}_{i v}=\left|\left[\partial_{\mu}-i\left(a_{i \mu}+\beta_{i \mu}\right)\right] \Phi_{i v}\right|^{2}+\cdots, \\
\mathcal{L}_{\theta}=\frac{i}{\pi} \beta_{1 \mu} \epsilon_{\mu \nu \lambda} \partial_{\nu} \beta_{2 \lambda}, \\
\mathcal{L}_{A}=i A_{i \mu} j_{i \mu} .
\end{gathered}
$$

Here, $\mu, \nu, \lambda, \ldots$ represent spacetime indices in $2+1$ dimensions. The $a_{i}, i=1.2$ are the usual dual-gauge fields of the vortex theory. The physical current $j_{i \mu}$ of the bosons $b_{1,2}$ is given as usual by 


$$
j_{i \mu}=\frac{1}{2 \pi} \epsilon_{\mu \nu \lambda} \partial_{\nu} a_{i \lambda}
$$

We have included external probe gauge fields $A_{i \mu}$ that couple to these currents. The $\beta_{i \mu}$ are "statistical" gauge fields that serve to impose the mutual statistics of the two vortex species through the mutual Chern-Simons term in $\mathcal{L}_{\theta}$. We have also tuned away a chemical potential that couples to the total boson number so that the effective action is relativistic. We relax that assumption shortly.

In passing, we note that recently related models of two species of bosons with mutual $\pi$ statistics have been studied numerically through Monte Carlo simulations [31]. The relevance of these models to the surface of the 3D boson topological insulator (and the related 2D boson integer quantum Hall transition) should give further impetus for such studies.

\section{Synthesis of the two approaches}

We now provide a synthesis of the results of the two approaches taken in Sec. IV. We rely closely on the results of Ref. [33] to provide two alternate field-theoretic representations of the theory described by Eq. (28). Rather than repeat the derivation from Ref. [33], we provide a physical description. The $\pi$ phase picked up when the vortex $\Phi_{1 v}$ goes around the vortex $\Phi_{2 v}$ suggests that $\Phi_{1 v}$ carries $1 / 2$ charge under the global U(1) symmetry associated with species 2 and vice versa [i.e., that $\Phi_{2 v}$ carries $1 / 2$ charge under the global $\mathrm{U}(1)$ of species 1]. However the $\pi$ phase is obtained for both charge $1 / 2$ and charge $-1 / 2$. We thus should expect that the vortex of either species carries fractional charge $\pm 1 / 2$ of the global $U(1)$ quantum number of the other species. This expectation is formalized by the derivation [33]. First, by performing a duality on one species (say, 1), we explicitly map to an easy-plane noncompact $C P^{1}$ model with the action given by Eq. (14).

If instead we had performed a duality transformation on species 2 , we would have obtained an equivalent action in terms of the fractionalized fields $\psi_{1 \pm}$ related to $b_{1}$ through

$$
b_{1}^{\dagger}=\psi_{1+}^{\dagger} \psi_{1-} .
$$

This action takes the form

$$
\begin{aligned}
\mathcal{L}_{2}= & \sum_{s}\left|\left(\partial_{\mu}-i \alpha_{1 \mu}-i \frac{s A_{1}}{2}\right) \psi_{1 s}\right|^{2}+\cdots \\
& +\frac{1}{2 \kappa_{1}}\left(\epsilon_{\mu \nu \lambda} \partial_{\nu} \alpha_{1 \lambda}\right)^{2}+\frac{i}{2 \pi} A_{2 \mu} \epsilon_{\mu \nu \lambda} \partial_{\nu} \alpha_{1 \lambda},
\end{aligned}
$$

with the physical $\mathrm{U}(1)$ current of the $b_{2}$ bosons given by $j_{2 \mu}=\frac{1}{2 \pi} \epsilon_{\mu \nu \lambda} \partial_{\nu} \alpha_{1 \lambda}$. Note the obvious similarity between Eq. (34) and Eq. (14) after interchange of the 1 and 2 labels. This similarity is a reflection of the self-duality of the easy-plane $\mathrm{NCCP}^{1}$ model first pointed out in Ref. [41]. This self-duality is obvious when both theories are
TABLE I. Symmetry properties of the $\psi_{1 \pm}, \psi_{2 \pm}$ fields. $q_{1,2}$ are the charges under the two $\mathrm{U}(1)$ symmetries associated with $b_{1,2}$, respectively. $n_{v 1,2}$ are the vorticities in the phase of $b_{1,2}$. They can also be viewed as the gauge charge for the coupling to the corresponding $\mathrm{U}(1)$ gauge fields. The last column gives the transformation under time reversal.

\begin{tabular}{lrrccc}
\hline \hline Field & $q_{1}$ & $q_{2}$ & $n_{v 1}$ & $n_{v 2}$ & $Z_{2}^{T}$ \\
\hline$\psi_{1+}^{\dagger}$ & $\frac{1}{2}$ & 0 & 0 & 1 & $\psi_{1-}$ \\
$\psi_{1-}^{\dagger}$ & $-\frac{1}{2}$ & 0 & 0 & 1 & $\psi_{1+}$ \\
$\psi_{2+}^{\dagger}$ & 0 & $\frac{1}{2}$ & 1 & 0 & $\psi_{2-}$ \\
$\psi_{2-}^{\dagger}$ & 0 & $-\frac{1}{2}$ & 1 & 0 & $\psi_{2+}$ \\
\hline \hline
\end{tabular}

obtained starting with the sigma model or the equivalent dual-vortex theory [Eq. (28)].

\section{Analysis of the surface field theory: Phase diagram, deconfined criticality, and fermionic vortices}

Having obtained a field-theoretic description of the surface states, we now analyze the phase diagram. The symmetry transformations summarized in Table I enable us to deduce the allowed perturbations to the actions above. A crucial allowed perturbation is "chemical-potential" terms that couple to the boson number $\mu_{1} n_{1}+\mu_{2} n_{2}$. Another crucial allowed perturbation is an interspecies bosontunneling term $-\lambda\left(b_{1}^{\dagger} b_{2}+\right.$ H.c. $)$. Let us first discuss the phase diagram when these terms are tuned to zero. Depending on the question being asked, we find it useful to use one or the other of the formulations provided above. For clarity of presentation, however, we use the theory in Eq. (14) when it is convenient.

$\mathcal{T}$-breaking states and the quantum Hall effect.Consider condensing just one of the vortex fields in Eq. (14):

$$
\left\langle\psi_{2+}\right\rangle \neq 0, \quad\left\langle\psi_{2-}\right\rangle=0 .
$$

Such a phase clearly breaks $T$-reversal symmetry, which interchanges the vortices. However, the phase is an insulator because the gauge-invariant combination $\left\langle\psi_{2+}^{*} \psi_{2-}\right\rangle=$ $\left\langle e^{i \phi_{2}}\right\rangle=0$. The transport properties of this phase are readily obtained by noticing that the combination $\alpha_{1}+\frac{A_{2}}{2}$ is gapped by the Higgs condensate. Therefore, at long wavelengths, we may set

$$
\alpha_{2} \approx-\frac{A_{2}}{2} .
$$

Furthermore, we may integrate out the field $\psi_{2-}$ in the Lagrangian equation (14). The effective long-wavelength Lagrangian for the external probe gauge fields then becomes

$$
\mathcal{L}_{\text {eff }}=-\frac{i}{4 \pi} A_{1 \mu} \epsilon_{\mu \nu \lambda} \partial_{\nu} A_{2 \lambda} .
$$

Defining the "charge" and "pseudospin" probe gauge fields $A_{c}=\frac{A_{1}+A_{2}}{2}, A_{s}=\frac{A_{1}-A_{2}}{2}$, we obtain 


$$
\mathcal{L}_{\text {eff }}=-\frac{i}{4 \pi}\left(A_{c \mu} \epsilon_{\mu \nu \lambda} \partial_{\nu} A_{c \lambda}-A_{s \mu} \epsilon_{\mu \nu \lambda} \partial_{\nu} A_{s \lambda}\right),
$$

which implies that the charge Hall conductivity $\sigma_{x y}=-1$ while the pseudospin Hall conductivity $\sigma_{x y}^{s}=1$. Taken together, the thermal Hall conductivity $\kappa_{x y}=0$. If, on the other hand, we had condensed $\psi_{2-}$ without condensing $\psi_{1+}$, we would have found the time-reversed partner with $\sigma_{x y}=1$ and $\sigma_{x y}^{s}=-1$.

Consider now adding symmetry-allowed perturbations to the action. The surface state described above is gapped and hence is unaffected by the chemical potential terms if they are weak. The interspecies tunneling term destroys conservation of pseudospin $\left(=n_{1}-n_{2}\right)$ and hence $\sigma_{x y}^{s}$ is no longer quantized. However, the electrical and thermal Hall conductivities continue to be well defined and have quantized values $\sigma_{x y}= \pm 1, \kappa_{x y}=0$. This result is exactly what we expect based on the general considerations of Sec. III.

Pictorially, these edge states may be understood by considering the bulk system on a solid sphere and assuming that $\psi_{1+}$ is condensed on the top hemispherical surface while $\psi_{1-}$ is condensed on the bottom hemispherical surface. Then, along the equator, there is a domain wall between the two kinds of surface quantum Hall states. At this domain wall, there are gapless $1 \mathrm{D}$ states identical to the edge of the 2D boson IQHE state. Specifically, there is one charged chiral mode corresponding to the jump $\Delta \sigma_{x y}=2$ across the domain wall and a counterpropagating neutral mode that carries the pseudospin. When interspecies tunneling is added, the quantization of the pseudospin Hall conductivity is not guaranteed, but the neutral edge mode is protected so long as charge is still conserved.

Superfluid state. Let us now consider $T$-reversalsymmetric phases. A simple option is

$$
\left\langle\psi_{2 s}\right\rangle=\psi_{0}
$$

independent of $s$. This state has $\left\langle b_{2}\right\rangle \neq 0$. The gauge field $\alpha_{2}$ is gapped by the Higgs $\psi_{2 \pm}$ condensate. Consider for a moment the situation in which the boson number is independently conserved for each species. Then this state breaks the global U(1) symmetry associated with $b_{2}$ but preserves the other global $\mathrm{U}(1)$ associated with $b_{1}$. We refer to it as $\mathrm{SF}_{1}$. If, on the other hand, both $\psi_{2 \pm}$ are gapped, then they may be integrated out to leave a Maxwell action for $\alpha_{1}$. Integrating out $\alpha_{1}$ then gives a Higgs mass for $A_{1}$ so that the global symmetry associated with $b_{1}$ is now broken. We call this $\mathrm{SF}_{2}$. These two phases are separated by a phase transition that is described by the putative critical point of the easy-plane $\mathrm{NCCP}^{1}$ field theory. In general, a chemical potential term can also be added that tunes the system away from the $\mathrm{NCCP}^{1}$ critical point. Apart from $\mathrm{SF}_{1}$ and $\mathrm{SF}_{2}$, we have the possibility of a phase with the coexistence of the two superfluid orders.
Inclusion of interspecies tunneling has a more dramatic effect. First, there is now no real distinction between the $\mathrm{SF}_{1}$ and $\mathrm{SF}_{2}$ phases so that the phase boundary between them disappears. Second, and more important, as the relative phase of $b_{1}^{\dagger} b_{2}$ can no longer wind, vortices in $b_{1}$ are bound to vortices in $b_{2}$. Note that $\psi_{1 \pm}$ are vortices in $b_{2}$ and $\psi_{2 \pm}$ are vortices in $b_{1}$. When we bind vortices in $b_{1}$ to vortices in $b_{2}$, the resulting vortices are created by fields,

$$
V_{s s^{\prime}}^{\dagger}=\psi_{1 s}^{\dagger} \psi_{2 s^{\prime}}^{\dagger},
$$

with $s, s^{\prime}= \pm$. Note that, for $s=-s^{\prime}, V_{s s^{\prime}}$ carries charge 0 under the single remaining global U(1) while, for $s=s^{\prime}$, it carries charge \pm 1 . Thus, the vortices no longer carry fractional charge. $V_{++}, V_{--}$can be obtained as a composite of the boson creation operator and the vortex $V_{+-}, V_{-+}$so that only the latter are "elementary." Furthermore, $V_{+-}$ can mix with $V_{-+}$due to the interspecies tunneling term. It follows that there is a unique elementary vortex $V \sim V_{+-}$ that carries charge 0. Also, under time reversal,

$$
V \rightarrow V^{\dagger} \text {. }
$$

Thus, in the presence of interspecies mixing, there is a unique vortex that does not transform projectively under the global symmetries. Does this fact invalidate our earlier analysis? In particular, can we now get a trivial insulator by condensing this vortex? The answer is "No." The point that we demonstrate below is that the effective action for the vortex $V$ in the superfluid phase is not the usual one; rather, it contains an extra Chern-Simons term. The presence of this Chern-Simons term has a convenient rough interpretation: It changes the statistics of the vortex to a fermion. A simple way to picture it is to describe it in terms of the vortex fields $\Phi_{v 1,2}$ in Eq. (28). The $\theta$ term in the sigma-model description means that the two vortices are mutual semions, and it follows that their bound state is a fermion.

To add some meat to this bare-bones picture, we start with Eq. (28). In the presence of interspecies tunneling, the individual vortex fields $\Phi_{1 v}, \Phi_{2 v}$ are confined, but a bound combination $\Phi_{c v}=\Phi_{1 v} \Phi_{2 v}$ survives. It is therefore necessary to reformulate the action in terms of $\Phi_{c v}$. We find it convenient to do so first even in the presence of the enlarged $\mathrm{U}(1) \times \mathrm{U}(1)$ symmetry and later to include the interspecies tunneling. Now we introduce another field $\Phi_{s v}=\Phi_{1 v} \Phi_{2 v}^{*}$. The resulting Lagrangian takes the form

$$
\begin{aligned}
\mathcal{L}= & \mathcal{L}\left[\Phi_{c v}, a_{+}+\beta_{+}\right]+\mathcal{L}\left[\Phi_{s v}, a_{-}+\beta_{-}\right] \\
& +\frac{i}{4 \pi} \beta_{+\mu} \epsilon_{\mu \nu \lambda} \partial_{\nu} \beta_{+\lambda}-\frac{i}{4 \pi} \beta_{-\mu} \epsilon_{\mu \nu \lambda} \partial_{\nu} \beta_{-\lambda} .
\end{aligned}
$$

Here, $a_{ \pm}=a_{1} \pm a_{2}, \beta_{ \pm}=\beta_{1} \pm \beta_{2} . a_{ \pm}$are the usual dual-gauge fields whose curl gives the charge and pseudospin current, respectively. The most interesting terms are the coupling to the gauge fields $\beta_{ \pm}$, which have 
self-Chern-Simons interactions. Including interspecies tunneling leads to linear confinement of $\Phi_{s v}$. The effective dual Landau-Ginzburg theory of the superfluid then has the usual form but with the additional Chern-Simons term, as promised.

Surface topological order-A wide variety of other phases is possible depending on the details of the surface interactions. For instance, a gapped topologically ordered $Z_{2}$ liquid is possible, accessed within the present formulation by condensing the paired vortex $\left(\psi_{2+} \psi_{2-}+\right.$ H.c. $)$ without condensing any other fields. In this situation, the full 3D system when placed on a solid torus has a groundstate degeneracy of 4 coming from the surface topological order. It is interesting to consider a bit further the properties of this state and its relationship with the superfluid state. In the $Z_{2}$ topologically ordered insulator, there is an unpaired vortex $\psi_{2+} \sim \psi_{2-}^{*}$ that survives as a gapped excitation and that carries physical boson charge $1 / 2$ of the $\mathrm{U}(1)_{2}$ global symmetry associated with the boson $b_{2}$. We refer to it as a 2 chargon. Following standard reasoning, this phase may equivalently also be understood as a paired condensate of $\psi_{1+} \psi_{1-}+$ H.c.. Thus, there is another gapped excitation corresponding to the field $\psi_{1+} \sim \psi_{1-}^{*}$ which, in the present context also carries charge $1 / 2$ of the physical boson $b_{1}$. We refer to it as the 1 chargon. These two chargons are mutual semions, as expected for $Z_{2}$ topological order. Note that they have bosonic self-statistics. In the presence of interspecies tunneling, a pair of 1 chargons can mix with a pair of 2 chargons. Both species of chargons continue to exist as independent excitations, but now they carry charge- $1 / 2$ of the remaining global U(1). Finally, the bound state of these two kinds of chargons is a fermion that does not carry fractional charge. We can take it to be charge neutral. It is convenient to regard this neutral fermion as the "vison," and the two kinds of bosonic chargons as the other two nontrivial quasiparticles expected for a $Z_{2}$ topological ordered state. These transformation laws are summarized in Table II.

Now let us consider the relationship of this topologically ordered state to the superfluid state discussed above, to clarify the nature of the vortices of the superfluid state. Coming from the superfluid side, the $Z_{2}$ topological state is obtained by condensing paired vortices. In the presence of

TABLE II. Symmetry properties of the topological excitations of a $Z_{2}$ gauge theory, realized on the surface of a 3D SPT phase with charge conservation and time-reversal symmetry $\left[\mathrm{U}(1) \rtimes Z_{2}^{T}\right]$, as for the topological insulator. The bosonic quasiparticles carry half charge but transform linearly under time reversal. Further details are in Appendix D.

\begin{tabular}{lll}
\hline \hline Excitation & Charge & $\mathcal{T}^{2}$ \\
\hline Boson 1 $(e)$ & $q=1 / 2$ & +1 \\
Boson 2 $(m)$ & $q=1 / 2$ & +1 \\
Fermion $(f)$ & $q=0$ & +1 \\
\hline \hline
\end{tabular}

interspecies tunneling, we have argued above that there is a unique vortex $V$. The unpaired vortex survives as a finiteenergy vison in this vortex-pair condensate. That this vison is a fermion ties in nicely with the observation that the superfluid vortex $V$ is conveniently regarded as a fermion. Thus, as the transition to this $Z_{2}$ insulator is approached, the vortex statistics becomes well defined and fermionic.

The topologically ordered phase provides a particularly simple perspective on why a trivial gapped paramagnet is not allowed. Generally, to go from a topologically ordered insulator to a trivial insulator, we must confine the topological quasiparticles. For a $Z_{2}$ gauge theory, this is done by condensing one of the three nontrivial kinds of quasiparticles (usually dubbed the electric, the magnetic, and their composite). For the $Z_{2}$ topological state that can appear at the surface of the SPT phase we are discussing, the electric and magnetic particles are both (half)-charged under the global U(1) symmetry, and their condensation breaks this symmetry. On the other hand, the neutral topological quasiparticle is a fermion and hence it cannot condense. At the same time, time reversal prevents one from altering the Chern number associated with this gapped fermion. Thus, we see clearly that a trivial gapped state obtained by confinement from the $Z_{2}$ topological state is not possible at the surface.

An interesting property of this $Z_{2}$ topological ordered state is that it realizes symmetry differently from strictly $2 \mathrm{D}$ systems. Such a strictly 2D gapped Abelian insulator may be described within the usual $K$ matrix formulation. For $Z_{2}$ topological order, $K=2 \sigma^{x}$. If both bosonic chargons carry charge $1 / 2$ as we argued, then the charge vector $\tau=(1,1)$. It is then easy to see that the resulting topological phase has nonzero electrical Hall conductivity and therefore must break time-reversal invariance. However, when realized at the surface of the 3D insulator, a time-reversal-symmetric $Z_{2}$ topological phase where both bosonic quasiparticles carry charge- $1 / 2$ is allowed. In Appendix $D$, we collect the properties of the $Z_{2}$ topological ordered state for the various SPT phases discussed here and show using the results of Ref. [43] that they all realize symmetry differently from what is allowed in strictly 2D systems.

While such interesting topologically ordered (or other even more exotic) states are allowed, they are not required: The surface could be in a superfluid or $T$-broken insulating quantum Hall state with no ground-state degeneracy. The most important conclusion, however, is that a trivial gapped insulating state that preserves all the symmetries and has no topological order is not possible on the surface. This realization is a key property of a symmetry-protected topological phase and is satisfied by our example.

Some remarks are now in order. We have constructed one topological phase, which, when the surface spontaneously breaks time-reversal symmetry, leads to a quantized magnetoelectric effect. The cohomology classification of Chen et al. [5] gives $Z_{2}^{2}$ so that there are three nontrivial 
states. Of the these three, one must have vanishing magnetoelectric effect since that is an additive quantity. We expect that this phase is simply the SPT phase associated with just the $Z_{2}^{T}$ symmetry discussed in Sec. VIA. The remaining phase is then obtained by combining the other two phases. In the particular example discussed in this section, the fact that there is a single $U(1)$ chargeconservation symmetry introduces additional terms that imply a deformation of the deconfined-criticality action. Despite this deformation, the degrees of freedom of this action provide the useful fields in terms of which the effective theory of the surface state may be described. We discuss other examples with different symmetries in subsequent sections.

\section{3D TOPOLOGICAL FIELD THEORIES}

To write a $3 \mathrm{D}$ theory of particles and their vortices, we choose to represent particle currents by $j^{\mu}=$ $\epsilon^{\mu \nu \lambda \sigma} \partial_{\nu} B_{\lambda \sigma} / 2 \pi$. The vortex lines, being loops in 3D space, sweep out a surface in spacetime defined by the two form $\gamma^{\mu \nu}$. Relating this two form to a vector potential $a$, whose curl is the location of the vortex loop, we define $\gamma^{\mu \nu}=\epsilon^{\mu \nu \lambda \sigma} \partial_{\lambda} a_{\sigma} / 2 \pi$. The quantization of boson particle number to integers implies that the charges that couple to this vector potential $a$ are quantized. (Equivalently, the dual vector potential is compact, i.e., defined only modulo $2 \pi$.) Clearly, gauge transformations $a_{\mu} \rightarrow a_{\mu}+\partial_{\mu} \chi$ and $B_{\mu \nu} \rightarrow B_{\mu \nu}+\left(\partial_{\mu} \alpha_{\nu}-\partial_{\nu} \alpha_{\mu}\right)$ do not change physical variables. Taking a particle around a vortex leads to a phase of $2 \pi$, which is captured by the minimal coupling $\mathcal{L}=$ $a_{\mu} j^{\mu}$, which may be rewritten as

$$
\mathcal{L}=\frac{1}{2 \pi} a_{\mu} \epsilon^{\mu \nu \lambda \sigma} \partial_{\nu} B_{\lambda \sigma}
$$

The right-hand side is often written as $\epsilon B \partial a / 2 \pi$ and called the "BF action." The unit coefficient in the action ensures that there is no topological order, i.e., that it is a unique ground state in the absence of surfaces [44] appropriate to the current discussion. Then, the theory above only states the obvious: that particles and their vortices have a mutual phase factor of $2 \pi$.

Let us briefly describe how this term arises from a microscopic theory of bosons. A lattice-regularized theory of bosons can be captured by a loop model of integervalued closed loops with Euclidean Lagrangian $\mathcal{L}_{b}=$ $\frac{1}{2 \rho} j_{\mu} j_{\mu}$. The integer constraint is implemented by summing over the auxiliary vector field $a_{\mu}$ that is an integer multiple of $2 \pi$. Now, the current $j_{\mu}$ takes real (rather than integer) values, and its divergence-free condition can be implemented by writing $j_{\mu}=\epsilon_{\mu \nu \lambda \sigma} \partial_{\nu} B_{\lambda \sigma} / 2 \pi$, where the two-form $B$ is also a real field. This gives

$$
\mathcal{L}_{b}^{E}=\frac{1}{8 \pi^{2} \rho}(\epsilon \partial B)^{2}+\frac{i}{2 \pi} \epsilon a \partial B,
$$

where the second term is the desired statistical interaction. (The factor of $i$ appears because of the Euclidean formulation.) However, at this point, $a$ is an integer (times $2 \pi$ ) field. One can softly introduce this constraint by assuming $a$ to be real but adding the cosine term $\Delta \mathcal{L}=$ $-\lambda \cos \left(\partial_{\mu} \phi-a_{\mu}\right)$, where we have utilized the fact that the longitudinal component can always be added to the gauge field [39]. The phase $\phi$ is actually the phase of the original bosons, and, when the bulk is insulating, the cosine is irrelevant since the bosons are gapped. Therefore, in the insulating phase, we may use Eq. (44) in which both $B, a$ fields are taken as real, with the caveat that charges are ultimately quantized. Further discussion of basic issues related to this theory is at the end of Appendix A.

As shown in Appendix A, surface states defined based on the BF-theory Eq. (44) are usual 2D bosonic modes that are not topologically protected. To encode a SRE topological phase, an additional term must be added, as shown below.

Based on the discussion on surface states, where a pair of bosonic fields were invoked, we consider two species of bosons to write a topological term. This step also follows the two-component $\mathrm{U}(1) \times \mathrm{U}(1)$-symmetric Chern-Simons approach for the 2D systems, which was found to be successful in describing 2D SRE topological phases [13]. Therefore, we introduce two $B$ fields that represent their conserved currents, and two $a$ fields that are vortices in these fields. Let us begin with the general case of $N$ species of bosons, with

$$
\mathcal{L}_{\mathrm{BF}}=\frac{1}{2 \pi} \sum_{I=1}^{N} \epsilon B_{I} \partial a_{I},
$$

where $\epsilon$ is the antisymmetric symbol, $I$ labels the distinct boson species, and indices have been suppressed.

Note that the apparently more general version is $\mathcal{L}_{B}=$ $\frac{Q_{I J}}{2 \pi} \epsilon B_{I} \partial a_{J}$ where $Q$ is a unimodular, i.e., $\operatorname{det} Q=1$, integer matrix, which ensures absence of topological order. However, this can be brought into the canonical form of Eq. (45) by redefining $B_{I}=\left[Q^{-1}\right]_{K I} B_{K}^{\prime}$. The transformation matrix $Q^{-1}$ is also an integer matrix, since $\operatorname{det} Q=1$ and the minors of an integer matrix are also integers.

Now, we can add an additional topological term to the action:

$$
\mathcal{L}_{3 D}=\mathcal{L}_{\mathrm{BF}}+\mathcal{L}_{\mathrm{FF}}, \quad \mathcal{L}_{\mathrm{FF}}=\frac{\Theta}{8 \pi^{2}} K_{I J} \epsilon \partial a_{I} \partial a_{J} .
$$

The action must be invariant under $\Theta \rightarrow \Theta+2 \pi$ to allow for addition of $2 \mathrm{D}$ layers at the surface. Therefore, the action defined on a closed 3D space should be invariant under the shift $\Theta \rightarrow \Theta+2 \pi$. We show in Appendix B that this condition fixes the entries $K_{I J}$ to be integers.

A stronger condition on $K$ can be applied as follows: Values of $\Theta$ that differ by $2 \pi$ simply correspond to different ways of terminating the surface. Hence, at a domain 
wall where $\Theta$ changes by $2 \pi$ at the surface, we demand that all excitations present are bosonic, which is the same as the requirement placed on $K$ matrices describing $2 \mathrm{D}$ SPT phases, i.e., that Det $K=1$ and that all diagonal entries are even integers.

The simplest choice of $K$ matrix with these properties is

$$
K=\left(\begin{array}{ll}
0 & 1 \\
1 & 0
\end{array}\right)
$$

We find that, for most of the 3D bosonic SPT phases that we are interested in, it suffices to consider this matrix. This choice is similar to the 2D situation [13], where the above $K$ matrix describes a large set of SPT phases that differ in the way symmetry is implemented.

\section{A. Two-component BF theory of bosonic topological insulator}

Let us specialize to the symmetries of the topological insulator $\mathrm{U}(1) \rtimes Z_{2}^{T}$ and consider a two-component theory with the simplest allowed $K$ matrix given by Eq. (47). Then we can write

$$
\begin{gathered}
\mathcal{L}_{\mathrm{tot}}=\mathcal{L}_{\mathrm{BF}}+\mathcal{L}_{\mathrm{FF}}+\mathcal{L}_{\mathrm{em}}, \\
\mathcal{L}_{\mathrm{BF}}=\frac{1}{2 \pi} \epsilon\left(B_{1} \partial a_{1}+B_{2} \partial a_{2}\right), \\
\mathcal{L}_{\mathrm{FF}}=\frac{\Theta}{4 \pi^{2}} \epsilon \partial a_{1} \partial a_{2} .
\end{gathered}
$$

(We discuss coupling to the external electromagnetic field $\mathcal{L}_{\text {em }}$ later in the paper.) Under time-reversal symmetry, we have

$$
\begin{aligned}
B_{I, 0 i} & \rightarrow-B_{I, 0 i}, \quad B_{I, i j} \rightarrow B_{I i j}, \\
a_{I, 0} & \rightarrow a_{I, 0}, \quad a_{I, j} \rightarrow-a_{I, j},
\end{aligned}
$$

where indices $i, j$ refer to spatial coordinates. The transformation of $B$ fields is obtained by relating them to the boson densities and currents, while the $a$ fields are chosen to transform such that the BF term is left invariant. Since both species $a_{I}$ transform in the same way under time reversal, we may conclude that $\Theta \rightarrow-\Theta$ under $Z_{2}^{T}$. A time-reversal-invariant bulk action can then be constructed for $\Theta=0, \pi$ (given the ambiguity in $\Theta$ modulo $2 \pi$ ). Of course, we pick $\Theta=\pi$ in the topological phase. While we have not derived this action, we have written the simplest possible topological theory that meets the general constraints required of SPT phases. We now proceed to show that this topological theory produces a surface with the same physical properties as predicted in the previous section. We study three different situations: First, we study the surface superfluid and determine the quantum numbers of vortices. Second, we investigate the electromagnetic response, particularly the magnetoelectric polarizability.
Third, we analyze the case in which time reversal is broken at the surface, in opposite ways, leading to a domain wall.

(i) Fractionally charged vortices.-Consider a surface of the topological phase at $z=0$ with $\Theta=\pi(0)$ for $z>0(z<0)$. Then the effective action at the surface arising from $\mathcal{L}_{\mathrm{FF}}$ is

$$
S_{\text {edge }}=\frac{1}{4 \pi} \int d t d x d y \epsilon^{z \alpha \beta \gamma} a_{1 \alpha} \partial_{\beta} a_{2 \gamma},
$$

where indices $\alpha, \beta, \gamma$ run over $t, x, y$, and the fields are evaluated at $z=0$ [45]. At the surface, we may replace $a_{I i}=\partial_{i} \phi_{I}$ (see Appendix A), where $i=x, y$. Consider now a surface superfluid of component $I=2$, with a vortex at the origin $x=y=0$. This configuration implies that $\phi_{2}$ winds around the origin or that $\left(\partial_{x} \partial_{y}-\partial_{y} \partial_{x}\right) \phi_{2}=$ $2 \pi n_{2}^{v} \delta(x) \delta(y)$, where we have allowed for a vortex of strength $n_{2}^{v}$. Substituting this for $\phi_{2}$ in Eq. (53), we have

$$
\mathcal{S}_{a}=\frac{1}{2} \int d t n_{2}^{v} \partial_{t} \phi_{1} .
$$

Similarly, a vortex in the field $\phi_{1}$ couples to the phase of $\phi_{2}$. Given the conjugate relation between number and phase, this implies that a vortex of strength $n_{2}^{v}$ in component $I=2$ carries charge $n_{2}^{v} / 2$ of component $I=1$. Thus we see that unit vortices in one bosonic field carry a half charge of the other field.

A different perspective on this result is obtained by thinking about the fate of "external" monopoles of the gauge fields $a_{1}, a_{2}$. These monopoles are sources for vortex lines of the two boson fields $b_{1}$ and $b_{2}$, respectively. Now, the well-known Witten effect implied by the $\Theta$ term tells us that a $2 \pi$ monopole in $a_{1}$ carries gauge charge $\frac{\Theta}{2 \pi}=\frac{1}{2}$ which couples to $a_{2}$, i.e., it carries charge $1 / 2$ of the global U(1) associated with $b_{2}$. Similarly, a $2 \pi$ monopole in $a_{2}$ carries a charge $1 / 2$ of the global $\mathrm{U}(1)$ associated with $b_{1}$. At the surface, this monopole creates a $2 \pi$ vortex of the corresponding boson, which is then seen to carry a half charge of the other boson, thereby recovering the result in the previous paragraph.

(ii) Electromagnetic response.-Since we have U(1) symmetry, we can couple to an external electromagnetic field and write the following terms:

$$
\mathcal{L}_{\mathrm{em}}=\frac{\epsilon}{2 \pi}\left(q_{1} B_{1} \partial A_{1}+q_{2} B_{2} \partial A_{2}\right)
$$

where $q_{I}$ is the charge on the Ith boson, given that the current of bosons $\epsilon \partial B_{I}=j$ couples minimally to $A_{I}$. If a single $\mathrm{U}(1)$ charge is present, then we identify $A_{1}=$ $A_{2}=A$. We see that this minimal coupling, along with Eq. (46), ensures that the charged vortex ends are also coupled to the external electromagnetic field.

To find the electromagnetic response, we integrate out the $B \mathrm{~s}$, and then the $a$. The first step gives $a_{I}=-q_{I} A_{I}$. Substituting this, we obtain 


$$
\mathcal{L}_{\text {em-response }}=\frac{\Theta}{4 \pi^{2}}\left[q_{1} q_{2}\right] \epsilon \partial A_{1} \partial A_{2} .
$$

Setting $\Theta=\pi, q_{1}=q_{2}=1$,

$$
\mathcal{L}_{\text {em-response }}=\frac{1}{4 \pi} \epsilon \partial A_{1} \partial A_{2},
$$

which indicates that ends of vortices in the condensate of the first species of bosons (which are induced by inserting $2 \pi$ flux in $A_{1}$ ) carry a half charge of the second species.

Finally, to obtain the response to the external electromagnetic field, we identify $A_{1}=A_{2}=A$, and then $\mathcal{L}_{\mathrm{em}}=$ $\frac{2 \pi}{4 \pi^{2}} \vec{E} \cdot \vec{B}$, which corresponds to a magnetoelectric polarizability of $\theta=2 \pi$, i.e., it is an odd multiple of $2 \pi$, as expected.

The theory above can also be derived using the hydrodynamic approach of [27]. Note that we could absorb the "FF" term in Eq. (48) [27] by redefining $B_{I} \rightarrow$ $B_{I}+\frac{\Theta}{4 \pi^{2}} K_{I J} \epsilon \partial a_{J}$. However, this redefinition leads to an additional electromagnetic coupling: $\frac{\Theta}{4 \pi^{2}} q_{I} K_{I J} \epsilon \partial A_{I} \partial a_{J}$. We still prefer to work with the original form since it is a field theory written in terms of internal fields, that is, it is well defined even in the absence of a conserved charge and external electromagnetic couplings.

(iii) Surface domain wall.-Finally, we consider an insulating surface in the $z=0$ plane, on which time reversal is broken in opposite ways for $y>0, y<0$. This leads to edge modes along the $x$ direction, localized near $z=y=$ 0 . This configuration is modeled with a spatially varying $\Theta$ field, where $\Theta(z>0)=0$, while $\Theta(z<0, y)=\pi \operatorname{sign}(y)$. Introducing this profile in $\mathcal{L}_{\text {tot }}$, with a gapped $B$ field on the surface, the edge theory is readily shown to be

$$
\begin{gathered}
\mathcal{S}_{\text {domain-wall }}=\frac{1}{2 \pi} \int d t d x\left[\partial_{x} \phi_{1} \partial_{t} \phi_{2}\right. \\
\left.+A_{0} \partial_{x}\left(q_{2} \phi_{1}+q_{1} \phi_{2}\right)+\cdots\right],
\end{gathered}
$$

where the first term defines the commutation relations of a regular Luttinger liquid, the second term identifies the coupling to the external field (assuming a gauge where $A_{x}=0$ ), and the dots refer to nonuniversal potential terms for the edge fields. This situation is identical to the edge state of the integer quantum Hall effect of bosons $[13,14]$ on setting $q_{1}=q_{2}=1$, which is also constant with the magnetoelectric polarizability of $\theta=\pi$ in this phase.

The general problem of deriving the above 3D field theory from microscopic models is left for future work. Next, we describe a bulk nonlinear sigma model, which in some ways may be considered a microscopic theory since it assumes additional ingredients, over and above the purely symmetry-group-based approach of the cohomology theory [5]. Therefore, instead of writing sigma models with topological terms, where the target manifold is the symmetry group, we allow the target manifold to be the four-sphere $S^{4}$, which assumes a particular microscopic representation.
However, since we are not concerned with classifying phases, but rather with providing physical examples, this additional assumption is convenient. This procedure is quite analogous to the common practice of considering crystalline band structures for free-fermion topological insulators, although they can (and, strictly speaking, should) be defined in the absence of translation symmetry [2].

\section{B. Bulk sigma model theory}

The bulk field theory discussed in the previous section is topological and has no bulk dynamical degrees of freedom. In this section, we describe a different bulk theory, one with dynamical boson fields, that gives rise to the topological effective field theory of the previous section in a disordered phase. This theory may thus be viewed as a field-theory realization of a model with a bosonic SPT phase in three space dimensions. This field theory takes the form of a $3+1$-dimensional nonlinear sigma model supplemented with a topological $\theta$ term. This form generalizes to three space dimensions the continuum field-theory model that realizes the $2 \mathrm{D}$ integer quantum Hall state of bosons.

Following the 2D example and the discussion in previous sections of this paper, we enlarge the symmetry of the boson system from U(1) to a larger symmetry group and then add perturbations to reduce to the symmetry of interest. For the construction of this section, it is extremely convenient to consider a generalization in which we first embed the $\mathrm{U}(1)$ symmetry into an $\mathrm{SO}(5)$ group. Consider therefore a five-component unit vector field $\hat{n}$. Later, we describe exactly how the physical symmetry $\left[\mathrm{U}(1) \times Z_{2}^{T}\right.$ or $\mathrm{U}(1) \rtimes Z_{2}^{T}$, etc.] are realized by the components of this field. For now, we write a continuum field theory for $\hat{n}$. On a closed spacetime manifold, say, the 4-sphere, the Lagrangian takes the form

$$
\begin{gathered}
\mathcal{L}=\mathcal{L}_{0}[\hat{n}]+\mathcal{L}_{\theta}[\hat{n}], \\
\mathcal{L}_{0}[\hat{n}]=\frac{1}{2 \lambda}\left(\partial_{\mu} \hat{n}\right)^{2}+\cdots, \\
\mathcal{L}_{\theta}[\hat{n}]=i \theta Q, \\
Q=\frac{1}{\Omega_{4}} \int d^{3} x d \tau \epsilon_{a b c d e} n_{a} \partial_{x} n_{b} \partial_{y} n_{c} \partial_{z} n_{d} \partial_{\tau} n_{e} \\
=\frac{3}{8 \pi^{2}} \int d^{3} x d \tau \operatorname{det}\left[\hat{n} \partial_{x} \hat{n} \partial_{y} \hat{n} \partial_{z} \hat{n} \partial_{\tau} \hat{n}\right],
\end{gathered}
$$

where $\Omega_{4}=\frac{8 \pi^{2}}{3}$ is the volume of the unit $4 \mathrm{D}$ sphere. $Q$ is the integer invariant corresponding to $\Pi_{4}\left(S^{4}\right)=Z$ and counts the number of times that spacetime configurations of the $\hat{n}$ field wrap around unit 4-sphere. Clearly, the theta term does not affect the bulk physics if $\theta=2 n \pi$ with integer $n$. (We note that, throughout this section, $\theta$ denotes 
the theta angle for the bulk sigma model; it should not be confused with the same symbol used previously for the electromagnetic response.) We are interested in disordered phases of this field theory where $\hat{n}$ is gapped in the bulk.

Consider a spatial domain wall between a state where $\theta=2 \pi$ and one where $\theta=0$. If this domain wall has a nontrivial surface state, then the $\theta=2 \pi$ theory describes an SPT phase in the bulk. Such a domain wall corresponds to a situation where $\theta$ varies spatially. To handle this situation, it is convenient to elevate $\theta$ to be a new dynamical field and to define a sigma model for a new sixcomponent unit vector field $\hat{\phi}$, defined by

$$
\hat{\phi}=\left(\begin{array}{c}
\cos \alpha \\
\hat{n} \sin \alpha
\end{array}\right)
$$

The field $\phi$ defines a map from spacetime (taken to be the 4D sphere $S^{4}$ ) to the 5D unit sphere $S^{5}$. Consider a field theory for $\hat{\phi}$ that includes (apart from the usual gradient terms) a WZW term (defined as usual as $2 \pi$ times the fraction of the volume of $S^{5}$ ) that is bounded by the hypersurface traced out by $\hat{\phi}$. Formally, let $\hat{\phi}(x, u)$ be a smooth extension of $\hat{\phi}(x)$ such that $\hat{\phi}(x, 0)=\hat{\phi}_{0}, \hat{\phi}(x, 1)=\hat{\phi}$. Then

$$
\mathcal{S}_{\mathrm{WZW}}[\hat{\phi}]=\frac{2}{\pi^{2}} \int_{\vec{x}, \tau} \int_{0}^{1} d u \operatorname{det}\left[\hat{\phi} \partial_{x} \hat{\phi} \partial_{y} \hat{\phi} \partial_{z} \hat{\phi} \partial_{\tau} \hat{\phi} \partial_{u} \hat{\phi}\right]
$$

Consider an extension where

$$
\hat{\phi}=\left(\begin{array}{c}
\cos \alpha(u) \\
\hat{n} \sin \alpha(u)
\end{array}\right),
$$

with $\alpha(0)=0, \alpha(1)=\alpha, \alpha(u)$ is independent of $\vec{x}, \tau)$, and $\hat{n}$ is independent of $u$. The determinant in $\mathcal{S}_{\mathrm{WZW}}$ is readily calculated and reduces to the theta term $\mathcal{L}_{\theta}[\hat{n}]$ for the $\hat{n}$ field, with

$$
\theta=\frac{16}{3} \int_{0}^{\alpha} d \alpha^{\prime} \sin ^{4} \alpha^{\prime}
$$

In particular, when $\alpha=0$, we get $\theta=0$, and when $\alpha=\pi$, we get $\theta=2 \pi$. Thus the WZW model for $\hat{\phi}$ with constant $\alpha=\pi$ describes the $3+1$-dimensional nonlinear sigma model for $\hat{n}$ at $\theta=2 \pi$.

To study a domain wall between $\theta=2 \pi$ and $\theta=0$ along, say, $z=0$, let $\alpha=\alpha(z)$ such that

$$
\begin{gathered}
\alpha(z \rightarrow-\infty)=0, \\
\alpha(z \rightarrow \infty)=\pi .
\end{gathered}
$$

We further assume that $\frac{d \alpha}{d z}$ is localized to within a short distance of $z=0$. To evaluate the WZW term in this configuration, it is convenient to use a different extension of the $\hat{\phi}$ field. Specifically, let

$$
\hat{\phi}=\left(\begin{array}{c}
\cos \alpha(z) \\
\hat{n}(\vec{x}, \tau, u) \sin \alpha(z)
\end{array}\right),
$$

with $\alpha$ now independent of $u, x, y, \tau$, and $\hat{n}(x, 0)=\hat{n}_{0}$, $\hat{n}_{x, 1}=\hat{n}(x)$. The determinant in $\mathcal{S}_{\mathrm{WZW}}$ is again readily evaluated and becomes

$$
\mathcal{S}_{\mathrm{WZW}}=\frac{2}{\pi^{2}} \int_{-\infty}^{\infty} d z \frac{d \alpha}{d z} \int_{x, u} \operatorname{det}\left[\hat{n} \partial_{x} \hat{n} \partial_{y} \hat{n} \partial_{z} \hat{n} \partial_{\tau} \hat{n} \partial_{u} \hat{n}\right]
$$

As $\frac{d \alpha}{d z}$ is localized at the domain wall at $z=0$, we can replace $\hat{n}$ in the integral by its configuration at $z=0$. The $z$ integral can now be performed and leads to

$$
\mathcal{L}_{\mathrm{WZW}}=\frac{3}{4 \pi} \int_{x, u} \operatorname{det}\left[\hat{n} \partial_{x} \hat{n} \partial_{y} \hat{n} \partial_{z} \hat{n} \partial_{\tau} \hat{n} \partial_{u} \hat{n}\right] .
$$

This is exactly the WZW term (at level 1 ) for the $\hat{n}$ field at the boundary. Thus the domain wall in question is described by a $2+1$-dimensional $\mathrm{SO}(5)$ nonlinear sigma model with a WZW term.

This field-theoretic result is very useful for constructing a bulk-sigma-model description of the SPT phases discussed in this paper. The simplest application is to bosons with symmetry $\mathrm{U}(1) \times Z_{2}^{T}$, discussed in detail in the next section. To illustrate this application, let us first introduce a $\mathrm{U}(1) \times \mathrm{SO}(3)$ anisotropy and write $\hat{n}=[\operatorname{Re} \psi, \operatorname{Im} \psi, \vec{N}]$, where, under the global U(1) symmetry, we choose $\psi \rightarrow$ $e^{i \epsilon} \psi$, but $\vec{N} \rightarrow \vec{N}$. Under time-reversal, we let $\psi \rightarrow$ $\psi^{*} \vec{N} \rightarrow-\vec{N}$. Finally, under the global $\mathrm{SO}(3)$ symmetry, $\vec{N}$ transforms as a vector while $\psi$ is invariant.

The level-1 WZW term plays the following crucial role [33] in this field theory: It implies that the vortex of the $\psi$ field transforms as spin-1/2 under the $\mathrm{SO}(3)$ rotation. Indeed, the five-component sigma model with global $\mathrm{SO}(3) \times \mathrm{U}(1)$ and $Z_{2}^{T}$ symmetries implemented in this way, and supplemented with a level-1 WZW term, precisely arises also as the theory of the deconfined critical point between Néel and VBS states in 2D. There, the spin- $1 / 2$ attached to the vortex captures the physical picture that a VBS vortex is a spinon.

For our present purposes, we need to further explicitly break the $\mathrm{SO}(3)$ symmetry while preserving time reversal. Then the $\vec{N}$ field is no longer a freely fluctuating variable. However, the crucial point is that, as the vortices of $\psi$ form a spinor, their Kramers degeneracy is preserved so long as $Z_{2}^{T}$ is preserved even if the full $\mathrm{SO}(3)$ is not present. This Kramers degeneracy is exactly the defining property of the surface theory of one of the SPT phases for bosons with $\mathrm{U}(1) \times Z_{2}^{T}$ symmetry described in the next section. We have thus obtained this surface theory from a bulk sigma model.

(i) Meaning of the theta term of the bulk sigma model.The sigma model description is useful because it suggests a route to obtaining a physical realization of this SPT phase. 
First, let us understand the meaning of the bulk $\theta$ term in this sigma model. As a topological term, it depends on the global configuration of the $\hat{n}$ field. In general, for a theory of $\vec{N}, \psi$ with $\mathrm{SO}(3) \times \mathrm{U}(1)$ symmetry, if there are no topological defects in either the $\vec{N}$ or the $\psi$ field, it is easy to see that the $\theta$ term vanishes. In $3 \mathrm{D}$, the $\vec{N}$ field admits point hedgehog defects, while the $\psi$ field admits vortex loops. The $\theta$ term implies that, during a process in which a hedgehog is taken around a vortex line, a phase $e^{i \theta}$ accumulates. For $\theta=2 \pi$, it follows that the hedgehog of $\vec{N}$ has charge 1 under the global $\mathrm{U}(1)$ symmetry associated with $\psi$. (This charge ensures that the hedgehog acquires phase $2 \pi$ when it moves around a vortex line.) Thus this kind of SPT phase may potentially be engineered by constructing a physical situation in which the hedgehog defect of a three-component order parameter is charged under the global U(1) symmetry of the bosons of interest.

(ii) Other SPT phases.-To describe the other SPT phases discussed above with the same symmetries, we must implement the symmetry differently. First, we consider a different situation, where the $\mathrm{SO}(3)$ vector has only $\mathrm{U}(1) \times Z_{2}^{T}$ symmetry. Then we write $\hat{n}=\left(\operatorname{Re} \psi_{1}, \operatorname{Im} \psi_{1}\right.$, $\left.\operatorname{Re} \psi_{2}, \operatorname{Im} \psi_{2}, N_{z}\right)$. Following the reasoning of the previous sections, we take both $\psi_{1}$ and $\psi_{2}$ to be charged under the global $\mathrm{U}(1)$ symmetry. For $\mathrm{U}(1) \times Z_{2}^{T}$, we take under time reversal $\psi_{1,2} \rightarrow \psi_{1,2}^{*}$ and $N_{z} \rightarrow-N_{z}$. If time reversal is preserved, then $\left\langle N_{z}\right\rangle=0$. In the presence of anisotropy that favors the $\hat{n}$ to have zero component of $N_{z}$, we may drop $N_{z}$ to obtain an effective field theory for the surface. Then the level- 1 WZW term for $\hat{n}$ becomes the familiar $\theta$ term at $\theta=\pi$ for the remaining four-components in the $2+1$-dimensional surface theory. To understand the bulk, note that, when there is $\mathrm{U}(1) \times Z_{2}^{T}$ anisotropy on an $\mathrm{SO}(3)$ vector, the vortex lines of the $\mathrm{U}(1)$ field $\psi_{2}$ come in two kinds, which are distinguished by the sign of $N_{z}$ in the core. The hedgehogs of the original $\vec{N}$ field are then domain walls within the cores of these vortices where the $N_{z}$ changes sign [46]. Thus the hedgehog must be regarded as a composite of two kinds of monopole sources for the two kinds of vortex lines. Formally, we may write the hedgehog creation operator $h^{\dagger}$ as

$$
h^{\dagger}=m_{2+}^{\dagger} m_{2-},
$$

where $m_{2 \pm}$ create the two kinds of monopole sources. Now the charge 1 of the hedgehog implied by the bulk $\theta$ term implies that these monopole sources are charged. Further, as $Z_{2}^{T}$ changes the sign of $N_{z}$, it interchanges the two monopole sources. It follows that the monopoles $m_{ \pm}^{\dagger}$ each carry charge $\pm 1 / 2$ of the $\psi_{1}$ field. This is precisely what is implied by the Witten effect as applied to the two-component BF + FF topological field theory of the previous section.

Exactly the same description can be provided for the boson topological insulator with symmetry $\mathrm{U}(1) \rtimes Z_{2}^{T}$. Then we consider anisotropy similar to that above with $\psi_{1,2}$ charged under the global U(1) symmetry, but we let $\psi_{1,2} \rightarrow \psi_{1,2}, N_{z} \rightarrow-N_{z}$ under $Z_{2}^{T}$. The rest of the discussion is identical to the one above.

This analysis establishes the connection between the bulk sigma model and topological field theory descriptions. Apart from giving an alternate perspective, we hope that the ideas of this section provide insights into physical realization of these SPT phases, a task we leave for the future.

\section{OTHER SYMMETRIES: TOPOLOGICAL PARAMAGNETS}

Now let us study various other symmetries that are particularly appropriate to quantum spin systems. By analogy with electronic topological insulators, SPT phases in quantum magnets may be christened "topological paramagnets." In particular, we highlight two cases: (i) $Z_{2}^{T}$ time-reversal symmetry. This case is the simplest symmetry that produces a topological phase, and we construct a nontrivial phase, thus indicating a $Z_{2}$ class. In the absence of a conserved charge, there is no quantized magnetoelectric effect. In the next section, we construct a separate topological phase with this symmetry but with chiral modes on a domain wall. (ii) $\mathrm{U}(1) \times Z_{2}^{T}$. This case corresponds physically to a timereversal invariant spin system in which the $z$ component of spin is conserved. Two nontrivial phases are constructed: The first has a quantized $\theta=2 \pi$ but the symmetry prohibits background charge, which allows us to sharply define statistics of vortices. The possibility of an exotic type of Bose liquid, the vortex metal, as a generic surface state is discussed. The second nontrivial phase has $\theta=0$. However, in this case, we show that a deconfined quantum critical action could emerge on tuning just a few parameters. We relegate to Appendix $\mathrm{C}$ a third symmetry, which is also readily analyzed: $\mathrm{U}(1) \rtimes Z_{2}$, for which we obtain $Z_{2}$ topological phases. This symmetry is of interest since it does not involve time-reversal symmetry.

\section{A. Symmetry $Z_{2}^{T}$}

We now consider the case of only time-reversal symmetry, both by analyzing the projective representations of surface vortices and by constructing bulk field theories.

Surface theory.-As usual, it is convenient to assume a slightly bigger symmetry to identify the relevant physics and then to break it down to the physical symmetry. Here it is sufficient to enlarge the symmetry to $\mathrm{U}(1) \times Z_{2}^{T}$ so that we may discuss vortices in a boson field $b_{1}^{\dagger}=e^{i \phi_{1}}$.

Let us first discuss the transformation of the $\phi_{1}$ field under time reversal. If this condition were like "chargephase," then, under time reversal, $\phi_{1} \rightarrow-\phi_{1}+\eta \pi$, where $\eta=0,1$. However, in such case, we would be able to pin $\phi_{1}$ for either value of $\eta$ by adding either a $\cos \phi_{1}$ or a $\sin \phi_{1}$ term. So this transformation does not correspond to an SPT phase boundary state, since the surface can be gapped in a trivial fashion without breaking symmetry. The other option is that $\phi_{1}$ transforms like the XY spin, 
i.e., $\phi_{1} \rightarrow \phi_{1}+\eta \pi$. In this case, for $\eta=1$, the term that can be added to the Lagrangian is $\cos \left(2 \phi_{1}+c\right)$, and the $\phi_{1}$ field cannot be gapped without breaking symmetry. Now we need to consider the transformation of the vortices because that can lead to a gapped state even if $\phi_{1}$ itself cannot be condensed. First, though, we note that the field $n_{1}$ conjugate to the phase $\phi_{1}$ transforms as $n_{1} \rightarrow-n_{1}$ under time reversal (i.e., like $S_{z}$ spin) to preserve the commutation relations.

Let us now discuss the transformation properties of the vortices under the remaining symmetry $Z_{2}^{T}$. What are the projective representations of the symmetry group $Z_{2}^{T}$ ? These are essentially the end states of a 1D topological phase with this symmetry. It is well known that there is a $Z_{2}$ classification of such phases and that the nontrivial phase is just the Haldane phase with gapless edge states that are spin $1 / 2$ objects. Therefore, the projective representation of $Z_{2}^{T}$ that we need is the following transformation of two vortex fields (just like spin 1/2) under time reversal, so $\psi_{2+} \rightarrow+\psi_{2-}, \psi_{2-} \rightarrow-\psi_{2+}$ or, more compactly,

$$
\Psi_{2} \rightarrow i \sigma_{y} \Psi_{2}
$$

Note that, if the vortex fields condense, they can condense either individually or simultaneously. In the individual case, the gauge-invariant operator $N^{z}=\left|\psi_{2+}\right|^{2}-\left|\psi_{2-}\right|^{2}$ takes on a nonzero expectation value. This object, under time reversal, transforms as $N^{z} \rightarrow-N^{z}$, so it breaks time reversal. If both fields condense simultaneously, then the gauge-invariant field $\psi_{2+}^{*} \psi_{2-}=e^{i \phi_{2}}$ acquires an expectation value. However, under time reversal, $e^{i \phi_{2}}$ transforms nontrivially as $\phi_{2} \rightarrow \phi_{2}+\pi$ (note $\phi_{1}$ and $\phi_{2}$ transform the same way) and cannot take on an expectation value without breaking time-reversal symmetry. (Both of these vortex fields are essentially spin operators.) A third option is that $\psi_{2 s}$ do not condense individually, but rather a pair condenses, which leads to a $Z_{2}$ topologically ordered state that does not break symmetry, making it also consistent with a topological surface state.

The effective theory for bosons at the edge consistent with this symmetry is

$\mathcal{L}_{e}=\sum_{s}\left|\left(\partial_{\mu}-i \alpha_{2 \mu}\right) \psi_{2 s}\right|^{2}+\frac{1}{2 \kappa} f_{2 \mu \nu}^{2}+\sum_{m}\left(\lambda_{m} V^{2 m}+\right.$ c.c. $)$,

where $V_{2 m}=e^{i 2 m \phi_{1}}$ is the $2 m$ monopole insertion operator, which is allowed once we break the U(1) symmetry to leave just the time-reversal invariance allowing even numbers of monopoles. Note that the background magnetic field $\left(\partial_{x} \alpha_{2 y}-\partial_{y} \alpha_{2 x}\right)=2 \pi n_{1}$ is odd under time-reversal symmetry and is not allowed. Also note, however, that the boson mixing terms $e^{i\left(\phi_{1} \pm \phi_{2}\right)}$ are allowed by symmetry. Other symmetry-allowed terms are the same as with $\mathrm{U}(1) \times Z_{2}^{T}$ symmetry, which is discussed following Eq. (80).

Surface $Z_{2}$ topological order and symmetry.-Just as we did in Sec. IV D, we find it extremely instructive to consider the question of why a trivial paramagnetic state is not allowed at the surface from the point of view of the $Z_{2}$ topological surface state. Here, of the three nontrivial topological quasiparticles, the two bosonic ones each transform as Kramers doublets under $Z_{2}^{T}$, i.e., $\mathcal{T}^{2}=-1$. They are simply the unpaired vortex of either $b_{1}$ or $b_{2}$. They have mutual semionic statistics. Their bound state is a $Z_{2}^{T}$ singlet $\left(\mathcal{T}^{2}=+1\right)$, but it is a fermion. To destroy the topological order, we must condense one of these nontrivial quasiparticles. However, when either of the two bosonic excitations condense, $Z_{2}^{T}$ is spontaneously broken. The fermion cannot condense, and time-reversal symmetry prohibits a nontrivial Chern number for fermions. Thus there is no possibility of a trivial paramagnet.

The cohomology classification [5] also produces one nontrivial SPT phase with this symmetry. Our analysis gives a direct understanding of the allowed surface structure of this phase. However, later we point out another distinct nontrivial SPT phase with $Z_{2}^{T}$ symmetry that appears to be beyond the cohomology classification.

$3 D$ bulk Lagrangian.-Consider the bulk Lagrangian

$$
\mathcal{L}_{\mathrm{BF}}=\frac{\epsilon}{2 \pi}\left(B_{1} \partial a_{1}+B_{2} \partial a_{2}\right)+\Theta \frac{\epsilon}{4 \pi^{2}} \partial a_{1} \partial a_{2} .
$$

The first term is invariant under time reversal if we assume $B_{I}^{0 i} \rightarrow B_{I}^{0 i}$ and $a_{I}^{i} \rightarrow a_{I}^{i}$ while $B_{I}^{i j} \rightarrow-B_{I}^{i j}$ and $a_{I}^{0} \rightarrow-a_{I}^{0}$ under time reversal. The transformation law for $B$ is obtained by assuming that it is connected to a conserved current that transforms like spin current. Thus, unlike Chern-Simons in 2D, the bulk action is naturally invariant under $T$. However, the second term changes sign if both $a_{I}$ transform in the same way. This observation fixes $\Theta$ to one of the two values 0 and $\pi$, yielding at least two phases. Since there is no conserved charge, there is no coupling to an external field. However, the "fractionalized" degrees of freedom at the ends of vortices are captured in this formalism.

It is relevant to note that phenomena previously used to distinguish topological phases do not apply for this symmetry. For example, the absence of a conserved charge does not allow us to define the magnetoelectric polarizability. Also, it turns out that the domain wall between opposite surfaces that break time-reversal symmetry does not carry gapless modes. Recall that, in free-fermion 3D topological insulators and class DIII topological superconductors, there are chiral edge modes on $T$-breaking domain walls. Also, for bosonic topological insulators, there is a nonchiral but protected domain wall mode. Here, however, the domain wall is nonchiral [as can be seen from the $K=\sigma_{x}$ matrix that enters in the second term of Eq. (77)], and, in the absence of a conserved charge, the oppositely propagating modes can acquire a gap. Nevertheless, the surface states are still special, and they either are gapless, break symmetry, or develop topological order. 


\section{B. Symmetry U(1) $\times Z_{2}^{T}$}

Here the $\mathrm{U}(1)$ can be interpreted as spin-rotation symmetry about $z$ axis. However, we often continue referring to the conserved quantity as charge. We construct two different topological phases (and the composition of these phases defines a third nontrivial phase), each of which is interesting for different reasons.

Surface theory of phase 1 and the deconfined criticality action.-Here we do not enlarge the symmetry. Let bosons $b_{1}^{\dagger}=e^{i \phi_{1}}$ be charged under the $\mathrm{U}(1)$ symmetry so that

$$
\begin{aligned}
& \phi_{1} \rightarrow \phi_{1}+\epsilon: \mathrm{U}(1), \quad n_{1} \rightarrow n_{1}: \mathrm{U}(1), \\
& \phi_{1} \rightarrow \phi_{1}+\pi: Z_{2}^{T}, \quad n_{1} \rightarrow-n_{1}: Z_{2}^{T} .
\end{aligned}
$$

Next, we consider the vortices $\Psi_{2}$ of the field $e^{i \phi_{1}}$ and specify their transform under the remaining time-reversal symmetry, which has a single projective representation:

$$
\Psi_{2} \rightarrow i \sigma_{y} \Psi_{2}: Z_{2}^{T} \text {. }
$$

Now, since $\psi_{2+}^{*} \psi_{2-} \sim e^{i \phi_{2}}$, we have $\phi_{2} \rightarrow \phi_{2}+\pi$ under time reversal. The effective field theory is written as

$$
\begin{aligned}
\mathcal{L}_{e}= & \sum_{\sigma}\left|\left\{\partial_{\mu}-i \alpha_{2 \mu}\right) \psi_{2 \sigma}\right|^{2}+\frac{1}{2 \kappa} f_{2 \mu \nu}^{2} \\
& \left.-\lambda\left[\left(\psi_{2+}^{*} \psi_{2-}\right)^{2}+\text { H.c. }\right]+\mathcal{V}\left(\left|\Psi_{2}\right|^{2}\right)\right\} .
\end{aligned}
$$

The second to the last term is $\cos 2 \phi_{2}$, which preserves time-reversal symmetry. The flux $\left(\partial_{x} \alpha_{2 y}-\partial_{y} \alpha_{2 x}\right) / 2 \pi=$ $n_{1}$ vanishes on average since the density $n_{1}$ changes sign under time reversal. No monopole insertion operators are allowed since changing the flux corresponds to inserting conserved $\mathrm{U}(1)$ charge. We note that this action is very similar to the easy-plane noncompact $C P_{1}\left(\mathrm{NCCP}_{1}\right)$ action, proposed [20] as the critical theory between a spin 1/2 easy-plane Néel antiferromagnet and valencebond-solid (VBS) order. The flux here is just the spin density, while the vortex bilinear $e^{i \phi_{2}}$ correspond to the VBS order. In contrast to the square lattice with fourfold rotation symmetry, here the square of the VBS order parameter is allowed, as on a rectangular lattice. An important distinction from previously discussed deconfined criticality is that here translation symmetry is not invoked.

Symmetry actually permits other terms in this action, for example, the linear derivative terms $\left(\partial_{t} \alpha_{2 i}-\partial_{i} \alpha_{20}\right)$, which correspond to electric fields (spin currents) in the ground state. Similarly, finite gauge charge is also allowed in the ground state, corresponding to finite vortex density, since vortices here do not break time-reversal symmetry. This finite vortex density introduces linear time-derivative terms in the action above. However, if we expand the symmetry to include a $Z_{2}$ that reverses the orientation of the $\mathrm{U}(1)$ rotation axis, i.e., that sends $n_{1} \rightarrow-n_{1}$ (which is a rotation by $\pi$ around the $S_{x}$ axis in spin notation), then the additional terms discussed here are prohibited, since both electric field and gauge charge are odd under this $Z_{2}$. Thus, for the topological surface state with symmetry $\left[\mathrm{U}(1) \rtimes Z_{2}\right] \times Z_{2}^{T}$, the field theory is given by Eq. (80). Parenthetically, we note that precisely this internal symmetry was also assumed in the original discussion of deconfined criticality in 2D quantum magnets with easyplane anisotropy [20]. Generically, either the bosons or the vortices are condensed, which implies that either U(1) symmetry or time-reversal symmetry is broken. However, if the critical point separating these states is stable to fluctuations, then one could tune a single parameter and access a deconfined critical point on the surface. It is at present unclear if this holds true for the theory in Eq. (80), which is an easy-plane $\mathrm{NCCP}_{1}$ with a twofold $\lambda$ anisotropy term. There is mounting evidence that the $\mathrm{SU}(2)$ symmetric $\mathrm{NCCP}_{1}$ model supports a stable quantum critical point. While initial studies were divided between continuous $[41,47]$ and weak first order [48,49], recent studies of quantum models seem to favor continuous transition [5052]. However, the situation is less clear with easy-plane anisotropy [41,53] and the $\lambda$ anisotropy term above. The connection to SPT surface states should provide additional motivation for further study.

Thus far, we have assumed translation invariance on the surface, but in fact only internal symmetries are required to define the phase. The presence of surface randomness that respects internal symmetries provides random variations in the local critical coupling. This random energy density term is known to be typically relevant at a quantum critical point [54], since it requires a rather stringent condition to be met, $\nu>1$ for irrelevance in a clean critical point in $d=2$. Here we emphasize a crucial difference from the realization of the deconfined critical theory in 2D quantum magnets. In that case, the presence of disorder leads to a random field that couples linearly to the VBS order parameter. In the spinon representation, this is a random monopole insertion term. Alternatively, in the dual vortex representation, this is a random term that couples to $\psi_{2+}^{*} \psi_{2-}$. This coupling is expected to be relevant at the clean deconfined critical point and might potentially lead to confinement at the resulting disordered fixed point. Thus it is not clear if the $\mathrm{NCCP}_{1}$ description is a useful one in the presence of disorder. In the present problem, however, a linear coupling to $\psi_{2+}^{*} \psi_{2-}$ remains forbidden even in the presence of disorder. The random energy terms, though relevant, are still not expected to lead to confinement by themselves. More dangerous potentially are random variations in the coupling $\lambda$. The fate of the disordered $\mathrm{NCCP}_{1}$ model in the presence of this particular kind of randomness remains to be investigated. In this context, it may be relevant to note that even the fate of the $3 \mathrm{D}$ fermionictopological-insulator surface states in the presence of disorder and interactions is also not a settled issue. It is currently unclear if one of the symmetries is spontaneously broken in the low-energy limit. If the symmetries are 
preserved, a critical metal with universal conductance is predicted [55].

As in the other examples, a surface state with $Z_{2}$ topological order that preserves all symmetries is allowed and is readily accessed by condensing paired vortices. For both phase 1 and phase 2 discussed in this subsection with symmetry $\mathrm{U}(1) \times Z_{2}^{T}$, the symmetry properties of the corresponding surface topological order is summarized in Appendix D.

Surface theory of phase 2 and vortex spin metal.-A different topological phase is accessed by enlarging the symmetry momentarily to $[\mathrm{U}(1) \times \mathrm{U}(1)] \times Z_{2}^{T}$. Now we can assume that a boson of species $1 b_{1}^{\dagger}=e^{i \phi_{1}}$ be charged under the first $U(1)$ symmetry and that it transforms exactly as in Eq. (78). Vortices in this boson field transform under the remaining $\mathrm{U}(1)$ and time-reversal symmetry as

$$
\begin{gathered}
\Psi_{2} \rightarrow e^{i \epsilon^{\prime} \sigma_{z} / 2} \Psi_{2}: \mathrm{U}(1), \\
\Psi_{2} \rightarrow i \sigma_{y} \Psi_{2}: Z_{2}^{T} .
\end{gathered}
$$

Now the effective theory at the surface is

$$
\mathcal{L}_{e}=\sum_{\sigma}\left|\left(\partial_{\mu}-i \alpha_{2 \mu}\right) \psi_{2 \sigma}\right|^{2}+\frac{1}{2 \kappa} f_{2 \mu \nu}^{2}+\mathcal{V}\left(\left|\Psi_{2}\right|^{2}\right),
$$

which has neither monopole insertion nor anisotropy terms due to the presence of conservation of separate boson species. There is no background flux due to time-reversal symmetry. However, by breaking down the symmetry to the single $\mathrm{U}(1)$ symmetry, one is allowed the following term $\cos \left(\phi_{1}-\phi_{2}\right)$, since both bosonic fields transform the same way under symmetry. Now $V_{1}^{*}=e^{i \phi_{1}}$ corresponds to a monopole insertion operator. Thus this is a composite operator, which, in the variables above, may be written as $\psi_{2+}^{*} \psi_{2-} V_{1}+$ H.c.. It breaks down the $\mathrm{U}(1) \times \mathrm{U}(1)$ symmetry to a single $\mathrm{U}(1)$ and leads to a binding of their vortices. If only the time-reversal symmetry is broken, then a quantized Hall effect results from the conserved spin. The discussion closely parallels that in Sec. .

However, if $\mathrm{U}(1)$ symmetry is broken, the surface is an $X Y$-ordered state of the spin system, and the vortices can be shown to be fermionic as in Sec. IV D. An advantage in the topological paramagnet compared to the bosonic topological insulator surface is the absence of background spin density that implies that the fermionic vortices move in zero background field. This constraint allows for a sharp definition of their statistics in terms of the Berry phase under exchange. Moreover, since the vortex density does not break time-reversal symmetry, generically, a finite vortex density is present in the ground state. In the $X Y$ ordered state, these vortices form a vortex solid, and their statistics is not very important. It is, however, extremely interesting to ask about the result of destroying the $X Y$ order by melting the vortex solid and proliferating the vortices. With Fermi statistics, the vortices form a Fermi surface that is coupled to the noncompact $\mathrm{U}(1)$ gauge field. The resulting state is a "vortex spin metal" - a compressible metallic phase of spins with many interesting properties. It is a gapless spin liquid with a vortex Fermi surface and is distinct from the more familiar 2D quantum spin liquids with a spinon Fermi surface. Ref. [56] proposed a very analogous vortex metal phase as an exotic possibility for a magnetic-field-driven quantum vortex liquid state in two space dimensions. There the magnetic field explicitly breaks time-reversal invariance. In contrast, the vortex spin metal obtained at the surface of the 3D topological paramagnet is a phase that preserves the defining $\mathrm{U}(1) \times Z_{2}^{T}$ symmetry. As with the other examples discussed in this paper, such a time-reversal-invariant vortex spin metal is presumably forbidden in strict 2D spin systems.

$3 D$ bulk theory.-For both phase 1 and phase 2, the 3D topological theories are identical; they differ only in the coupling of the conserved charge to the external field:

$$
\begin{gathered}
\mathcal{L}_{\text {tot }}=\mathcal{L}_{\text {topo }}+\mathcal{L}_{\text {em }}, \\
\mathcal{L}_{\text {topo }}=\frac{1}{2 \pi} \epsilon B_{I} \partial a_{I}+\Theta \frac{\epsilon}{4 \pi^{2}} \partial a_{1} \partial a_{2} .
\end{gathered}
$$

Under the $Z_{2}^{T}$ symmetry, $a_{1 i} \rightarrow a_{1}$, and $a_{2 i} \rightarrow a_{2 i}$ while their 0 components change sign. Thus the "axion" field $\Theta$ must be odd under $Z_{2}$, so the action as a whole is invariant. This property allows us to fix $\Theta=0, \pi$, and, of course, we pick the latter value in the topological phase. In general, in a 3D topological phase protected by time reversal, both fields should transform in the same way.

Now phase 1 has a single charged boson, $\phi_{1}$ :

$$
\mathcal{L}_{\mathrm{em}}^{\text {phase } 1}=\frac{1}{2 \pi} \epsilon B_{1} \partial A .
$$

The bulk theory predicts that $\theta=0$, i.e., no magnetoelectric effect for this phase.

However, for phase 2, both bosons are charged, so

$$
\mathcal{L}_{\mathrm{em}}^{\text {phase } 2}=\frac{1}{2 \pi} \epsilon\left(B_{1}+B_{2}\right) \partial A,
$$

and the bulk theory predicts $\theta=2 \pi$ magnetoelectric effect for this phase.

\section{3D $E_{8}$ PHASE WITH HALF-QUANTIZED SURFACE THERMAL HALL EFFECT AND MISCELLANEOUS COMMENTS}

Thus far, we have based our discussion of novel 3D SPT phases on the 2D $K=\sigma_{x}$ matrix. When a conserved charge is present, these phases often lead to a quantized magnetoelectric effect, or, equivalently, to a half-quantized surface Hall effect. On general grounds, one may expect additional phases based on the fact that thermal transport can also be quantized. In these phases, chiral modes are expected at the domain walls between opposite-symmetrybreaking regions, which lead to the quantized thermal Hall conductance. In this section, we provide a possible field-theoretic description of such a phase. 
Recall that, in a 2D system, the combination $\kappa_{x y} / T=$ $\nu_{T} \frac{\pi^{2} k_{B}^{2}}{3 h}$ is quantized, where $\kappa_{x y}$ is the thermal Hall conductance and $T$ is temperature, in the limit of $T \rightarrow 0$. Here, $\nu_{T}$ counts the number of chiral boson modes at the edge. For bosons with SRE in $d=2$, it is known that the quantization takes values $\nu_{T}=8 n$ that are multiples of 8 times quantum of thermal conductance. Anything else leads to topological order. These states are based on the $K$ matrix of the Kitaev $E_{8}$ state [13]:

$$
K^{E_{8}}=\left(\begin{array}{cccccccc}
2 & -1 & 0 & 0 & 0 & 0 & 0 & 0 \\
-1 & 2 & -1 & 0 & 0 & 0 & 0 & 0 \\
0 & -1 & 2 & -1 & 0 & 0 & 0 & -1 \\
0 & 0 & -1 & 2 & -1 & 0 & 0 & 0 \\
0 & 0 & 0 & -1 & 2 & -1 & 0 & 0 \\
0 & 0 & 0 & 0 & -1 & 2 & -1 & 0 \\
0 & 0 & 0 & 0 & 0 & -1 & 2 & 0 \\
0 & 0 & -1 & 0 & 0 & 0 & 0 & 2
\end{array}\right) .
$$

One may utilize this fact to construct the following 3D SPT phase. Assume time-reversal symmetry $Z_{2}^{T}$ is present. Consider the $3 \mathrm{D}$ theory given by

$$
\mathcal{L}=\frac{1}{2 \pi} \sum_{I=1}^{8} \epsilon B_{I} \partial a_{I}+\Theta \sum_{I, J} \frac{K_{I J}^{E_{8}}}{8 \pi^{2}} \epsilon \partial a_{I} \partial a_{J} .
$$

As long as all the fields $a_{I}$ transform the same way under time-reversal symmetry, the coefficient $\Theta$ is quantized to $\Theta=0, \pi$. The latter leads to a topological phase. If timereversal symmetry is preserved in the bulk but broken on the surface, it is readily seen that each domain has thermal Hall conductivity $\nu_{T}= \pm 4$, and a domain wall between opposite domains has the eight chiral edge states of the 2D theory specified by the $K$ matrix in Eq. (87).

It is likely that such a state lies beyond cohomology classification since we have already identified a phase based on $K=\sigma_{x}$ in this symmetry class that exhausts the set of states predicted by cohomology theory [5]. A question that is relevant in this context is whether the field theory above can be realized within a lattice model. One difference from the other topological phases we have described based on the $K=\sigma_{x}$ FF term is that, in those cases, a lattice regularization of the field theory can be readily envisaged since it involves a Berry phase for the product of electric and magnetic fields representing two different species of vortices $\epsilon F_{1} F_{2} \rightarrow E_{1} \dot{B}_{2}+\vec{E}_{2} \cdot \vec{B}_{1}$. This term is naturally discretized by assuming that the corresponding vector potentials live on the links of the direct lattice and the dual lattice. However, the diagonal entries of the $K^{E_{8}}$ matrix above lead to terms that are not obviously compatible with a lattice. Whether this fact imposes an additional constraint on possible phases is an important open question. If, indeed, the phase described above is physically admissible, then it remains to be clarified if the additional states lead to a $Z_{2}$ or a $Z$ extension (assuming just timereversal symmetry). We leave these questions to future study. In this context, it may be relevant to note that the analogous free-fermion phases are topological superconductors in 3D protected by time-reversal symmetry (Class DIII), which are classified by integers. On the other hand, one may ask, What are the properties of the topologically ordered surface state that is fully symmetric? We conjecture that a candidate state is a $Z_{2}$ topological ordered state in which all three nontrivial excitations are fermionic and have $\pi$ mutual statistics. Such a state when realized in 2D is given by the $K$ matrix:

$$
K^{\mathrm{SO}(8)}=\left(\begin{array}{cccc}
2 & -1 & -1 & -1 \\
-1 & 2 & 0 & 0 \\
-1 & 0 & 2 & 0 \\
-1 & 0 & 0 & 2
\end{array}\right),
$$

which is the Cartan matrix of $\mathrm{SO}(8)$. This state, when realized in 2D, has four chiral edge states and hence must break time-reversal symmetry. However, it may appear on the surface of a 3D SPT phase, protected by time-reversal symmetry.

\section{CONCLUSIONS}

In summary, we highlight the remarkable similarities between free fermion topological insulators and the bosonic interacting topological phases described here. In the former case, the surface is gapped only on breaking one of the defining symmetries of charge conservation or timereversal symmetry. Then, the resulting ordered phase also possesses unusual properties. For example, when charge conservation is destroyed by a superconducting surface, the vortices carry a Majorana zero mode. Similarly, for the bosonic topological insulator with the same symmetries, breaking charge conservation at the surface leads to fermionic vortices (albeit without an attached Majorana zero mode). On the other hand, breaking just time-reversal symmetry leads for the fermionic case to a quantized magnetoelectric effect of $\theta=\pi$, whereas, for bosonic TIs in the same situation, the same response is quantized but at $\theta=2 \pi$. The fully symmetric surface of the fermionic TI, from which these conclusions can be readily derived, is a Dirac dispersion of free fermions. We propose that the analog for bosons is the deconfined quantum critical action, which describes a putative gapless state from which, on being subjected to various perturbations, realizes different ground states of the surface. It is also relevant to note that bosonic analogs of topological superconductors exist where domain walls between regions of opposite time-reversalsymmetry breaking carry gapless chiral modes.

It is interesting to further highlight the particular case of spin systems. The spin analogs of topological insulatorsthe topological paramagnets-may potentially be the most 
important realization in solid-state systems of the class of phases we have described. The surface of the topological paramagnet either spontaneously breaks symmetry or is in a quantum spin liquid state that is not allowed to exist in strict two dimensions with the same symmetry. We have discussed examples of such quantum spin liquids with surface topological order or with exotic gapless excitations. Our work raises fascinating questions on what kinds of spin liquids with symmetry are actually allowed to exist in strictly 2D systems that should be of direct importance to studies of 2D quantum magnetism.

There are several open questions for future work. Clearly, a central question is whether there are microscopic models, or perhaps even experimentally relevant systems, that could realize these phases. The bulk-sigma-model field theories may provide useful guidance in searching for such realizations. One route to accessing SPT phases in two dimensions is to start with a fractionalized phase and confine the fractionalized excitations. Our analysis suggests that a similar route may also be possible in three dimensions by starting with a fractionalized phase with emergent deconfined $\mathrm{U}(1) \times \mathrm{U}(1)$ gauge fields if these are confined by condensation [57] of mutual dyons [where a monopole of one $\mathrm{U}(1)$ gauge field is bound to particles that carry gauge charge of the other gauge field]. Exploring this possibility might also suggest physical realizations of the 3D SPT phases. A more formal question is whether one can push the field-theoretic descriptions of this paper to obtain all possible SPT phases in 3D, which could shed light on the way in which the chiral phases augment the cohomology characterization. The $3 \mathrm{D} \mathrm{BF}+\mathrm{FF}$ theories seem a convenient tool for capturing bosonic SPT phases. However, general constraints on the form of such theories are at present unclear.

\section{ACKNOWLEDGMENTS}

A. V. thanks Ari Turner and especially Yuan-Ming Lu for stimulating discussions and collaborations on related topics, and acknowledges support from NSF Contract No. DMR-1206728. T.S. thanks Liang Fu, Michael Levin, Chong Wang, Z. Gu, and Xiao-Gang Wen. T. S. was supported by NSF Contract No. DMR-1005434. We both thank Matthew Fisher for scintillating discussions and encouragement and for stimulating discussions on dyon condensation in 3D gauge theories as a route to describing exotic phases. This material is based on work supported in part by the National Science Foundation under Grant No. PHYS-1066293 and the hospitality of the Aspen Center for Physics. We also thank the Perimeter Institute for Theoretical Physics, and the Kavli Institute for Theoretical Physics where parts of this work were undertaken. This work was partially supported by Simons Foundation Nos. 229736 (T. S.) and 231377 (A. V.). On completing this work, we became aware of other studies that have some overlap with the present work $[58,59]$.

\section{APPENDIX A: 3D BF THEORY-SURFACE STATES AND EM RESPONSE}

As a warmup, let us recall the derivation of the edge states of a Chern-Simons theory in 2D [3]. We specialize to the $K=\sigma_{x}$ Chern-Simons theory:

$$
\mathcal{L}_{\mathrm{CS}}=\frac{\epsilon^{\mu \nu \lambda}}{2 \pi} a_{\mu}^{1} \partial_{\nu} a_{\lambda}^{2}
$$

Note that gauge invariance at the surface can be ensured by working in the gauge $a_{0}=0$. This condition implies $d a^{I}=0$ so $a_{i}^{I}=\partial_{i} \phi_{I}$ and gives the edge Lagrangian

$$
\mathcal{L}=\frac{1}{2 \pi} \partial_{x} \phi_{1} \partial_{\tau} \phi_{2}
$$

leading to the usual Kac-Moody commutation relations.

The edge dynamics originates from other terms. For example, we can add a Maxwell term to the original action $\left(\partial_{\mu} a_{\nu}^{I}-\partial_{\nu} a_{\mu}^{I}\right)^{2}$. The only low-derivative term that appears at the edge is from $\partial_{y} a_{x}^{I}$. Substituting the edge field $a_{x}^{I}=\partial_{x} \phi^{I}$ and noticing that the derivative perpendicular to the edge (i.e., along $y$ ) picks up only the confining wave function of the edge states, we are led to $\partial_{y} a_{x}^{I} \propto \partial_{x} \phi^{I}$. This reasoning gives potential terms

$$
\mathcal{L}_{1}=\rho\left[\left(\partial_{x} \phi_{1}\right)^{2}+\left(\partial_{x} \phi_{2}\right)^{2}\right] .
$$

Note that the pair of fields $\phi_{1}, \phi_{2}$, which are canonically conjugate Eq. (A2), is like any regular 1D Luttinger liquid. The special physics of SPT phases arises from the fact that the fields can transform under the symmetry in ways that a 1D system cannot. For example, in the U(1)-protected integer quantum Hall phase of bosons, the transformation law of the first nontrivial phase is $\phi_{i} \rightarrow \phi_{i}+\epsilon$. In other words, both fields transform under the charge rotation, which leads to protected edge states. By analogy, it appears that we should find that the surface of a $3+1 \mathrm{D}$ SPT phase of bosons is a regular 2D bosonic system, apart from application of symmetries. Indeed, we show below that this is the surface state of the $3+1 D$ BF theory.

Let us begin with the following 3D Lagrangian:

$$
\mathcal{L}_{\mathrm{BF}}=\frac{\epsilon^{\mu \nu \lambda \sigma}}{2 \pi} B_{\mu \nu} \partial_{\lambda} a_{\sigma} .
$$

Here, a bosonic current has been written as $j^{\mu}=$ $\epsilon^{\mu \nu \lambda \sigma} \partial_{\nu} B_{\lambda \sigma} / 2 \pi$, and $\partial \wedge a$ represents the vortex loops.

To derive surface properties, again we see that the nondynamical parts of the Lagrangian implement the constraint: $\epsilon_{i j} \partial_{i} a_{j}=0$ and $\epsilon_{i j k} \partial_{i} B_{j k}=0$. One can solve this expression to obtain $a_{i}=\partial_{i} \phi$ and $B_{i j}=\epsilon^{i j} \partial_{i} \alpha_{j}$. We take the gauge $B_{0 i}=a_{0}=0$. The topological part of the edge Lagrangian is computed next. [The edge is taken to be perpendicular to $z$, and we use the indices $a, b=(x, y)$.]

$$
\mathcal{L}=\frac{\epsilon^{a b}}{2 \pi} \partial_{a} \alpha_{b} \partial_{\tau} \phi .
$$


Now, for the dynamics, once again we introduce Maxwell terms in the bulk: $(d a)^{2}$ and $(d B)^{2}$. Again, the ones that survive with low derivatives have $\partial_{z}$ acting on them. The terms that appear are

$$
\mathcal{L}_{1}=\rho_{1}\left[\left(\partial_{x} \phi\right)^{2}+\left(\partial_{y} \phi\right)^{2}\right]+\rho_{2}\left[\left(\partial_{x} \alpha_{y}-\partial_{y} \alpha_{x}\right)^{2}\right] .
$$

Thus our boundary Lagrangian is (given $\mathcal{S}=\int d x d y d \tau \mathcal{L}_{e}$ )

$$
\begin{aligned}
\mathcal{L}_{e}= & \frac{\epsilon^{a b}}{2 \pi} \partial_{a} \alpha_{b} \partial_{\tau} \phi+\rho_{1}\left[\left(\partial_{x} \phi\right)^{2}+\left(\partial_{y} \phi\right)^{2}\right] \\
& +\rho_{2}\left[\left(\partial_{x} \alpha_{y}-\partial_{y} \alpha_{x}\right)^{2}\right] .
\end{aligned}
$$

One interpretation of this Lagrangian is that of a photon on a 2D surface where the Gauss law constraint has been solved, i.e., $2+1 \mathrm{D}$; the Gauss law $\partial_{x} E_{x}+\partial_{y} E_{y}=0$ can be solved by writing $E_{a}=\epsilon_{a b} \partial_{b} \phi / \pi$. Then the term that leads to canonical quantization of electric fields $E_{a} \partial_{\tau} \alpha_{a}$ is now replaced by $\frac{1}{\pi} \partial_{\tau} \phi\left(\partial_{x} \alpha_{y}-\partial_{y} \alpha_{x}\right)$, the first term in $\mathcal{L}_{e}$. The Hamiltonian of the Maxwell theory $\rho_{1}\left[E_{x}^{2}+E_{y}^{2}\right]+$ $\rho_{2}\left(\partial_{x} \alpha_{y}-\partial_{y} \alpha_{x}\right)^{2}$ is the term written above in the Lagrangian.

Although Refs. $[25,26]$ follow a rather similar derivation, they interpret the theory above as the bosonized description of a $2+1 \mathrm{D}$ Dirac fermion. This interesting speculation does not appear to be compatible with the wellknown fact that the theory described by Eq. (A6) is dual to a $2+1 \mathrm{D}$ theory of bosons. Explicitly, this can be seen as follows: Since $\epsilon^{a b} \partial_{a} \alpha_{b} / 2 \pi$ is conjugate to the phase $\phi$, we denote it by $\Pi_{\phi}=\epsilon^{a b} \partial_{a} \alpha_{b} / 2 \pi$ and use this field to write the Hamiltonian of the surface theory as

$$
H=4 \pi^{2} \rho_{2} \Pi_{\phi}^{2}+\rho_{1}(\nabla \phi)^{2} .
$$

This Hamiltonian is just the theory of a boson in the two spatial dimensions of the surface (as expected, since we began with a bosonic theory).

It is useful to catalog the connection between the dual descriptions. The monopole insertion operator in the surface electrodynamics is $e^{i \phi}$ and actually corresponds to the insertion of particles. The other excitations, "gauge charges" of the gauge theory, are the ends of vortices of the $3 \mathrm{D}$ bulk and are point particles on the surface. Here they behave like charges in the 2D electrodynamics, since vortices of the $\phi$ field are equivalent to violating Gauss law for the electric field. Also, since Curl $a \rightarrow \operatorname{Curl} \nabla \phi$, they correspond to the ends of the 3D vortices. The vortex insertion operator is of course a nonlocal object, which reflects the fact that one cannot insert a gauge-charged particle in the bulk without changing the gauge fields everywhere. The 2D surface is gapped either by monopoles $e^{i \phi}$ or by vortex condensation (Higgs mechanism). However, symmetry may forbid these, leading to SPT phases.

Let us briefly review some questions that arise in the context of the Lagrangian (A3). One can add terms such as $\mathcal{L}_{1} \sim(\epsilon \partial B)^{2}$ and $\mathcal{L}_{2} \sim\left(\partial_{\mu} a_{\nu}-\partial_{\mu} a_{\nu}\right)^{2}$, which are local and respect symmetries. Integrating out $B$ now appears to give the "Higgs" term $a_{\mu}^{2}$. However, it is readily seen that this still describes an insulator by coupling the charge to an external electromagnetic potential $A$ via $\epsilon A \partial B / 2 \pi$. Now integrating the fields $B$ essentially enforces $a \sim A$, which when substituted into $\mathcal{L}_{2}$ simply produces a Maxwell action for the external field: approximately $\left(\partial_{\nu} A_{\mu}-\partial_{\mu} A_{\nu}\right)^{2}$, as expected for an insulator. A 3D topological EM response appears in other cases where an FF term is present, by the same substitution.

\section{APPENDIX B: $\Theta$ PERIODICITY IN MULTICOMPONENT BF THEORY}

In this appendix, we prove the $2 \pi$ periodicity of $\Theta$ for the multicomponent BF theory. The Lagrangian is

$\mathcal{L}=\frac{1}{2 \pi} a_{\mu}^{I} \epsilon_{\mu \nu \lambda \kappa} \partial_{\nu} B_{\lambda \kappa}^{I}+\frac{\Theta}{8 \pi^{2}} K^{I J} \epsilon_{\mu \nu \lambda \kappa} \partial_{\mu} a_{\nu}^{I} \partial_{\lambda} a_{\kappa}^{J}$.

Summation is implicit over repeated component indices $I, J$. The crucial second term, when expressed in terms of the electric fields $\vec{e}^{I}$ and the magnetic fields $\vec{b}^{I}$, takes the form

$$
\frac{\Theta}{8 \pi^{2}} \sum_{I} K^{I I}\left[\left(2 \vec{e}^{I} \cdot \vec{b}^{I}\right)+\sum_{J>I} K^{I J} 2\left(\vec{e}^{I} \cdot \vec{b}^{J}+\vec{e}^{J} \cdot \vec{b}^{I}\right)\right] .
$$

Consider the theory on a closed three manifold such as a 3 -torus of size $L \times L \times L$. Through one cycle, say, the $x y$ cycle, slowly insert $2 \pi n_{I}$ magnetic flux of species $I$ at a rate $\frac{d \Phi_{I}}{d t}$. This operation leads to a bulk electric field along the $z$ direction:

$$
e_{z}^{I}=\frac{1}{L} \frac{d \phi_{I}}{d t} .
$$

Next, slowly turn on $2 \pi m_{I}$ flux of $b_{z}^{I}$ in the bulk so that

$$
b_{z}^{I}=\frac{2 \pi m_{I}}{L^{2}} .
$$

The quantum amplitude for these processes is given by the $\Theta$ term in the action and takes the form

$$
\begin{aligned}
& e^{i\left(\Theta / 4 \pi^{2}\right) \int d t L^{3}\left(2 \pi / L^{2}\right)\left\{\sum_{I}\left(K^{I I} m_{I}\left(d \Phi_{I} / d t\right)+\sum_{J>I} K^{I J} m_{J}\left(d \Phi_{I} / d t\right)\right\}\right.} \\
& =e^{i \Theta \sum_{I}\left(K^{I I} n_{I} m_{I}+\sum_{I \neq J} K^{I J} n_{I} m_{J}\right)} .
\end{aligned}
$$

For some particular pair $I, J$, choose $n_{I}=1, m_{J}=1$, and all other $n_{I^{\prime}}=m_{J^{\prime}}=0$. Then the amplitude simply becomes $e^{i \Theta K^{I J}}$. If all the elements $K^{I J}$ are integers, it follows that $\Theta$ is periodic under a $2 \pi$ shift.

\section{APPENDIX C: OTHER SYMMETRIES-U(1) $\rtimes \boldsymbol{Z}_{\mathbf{2}}$}

Here the $U(1)$ can be interpreted as spin-rotation symmetry about the $z$ axis, while the $Z_{2}$ is spin rotation by 180 degrees about the $x$ axis. 
Surface theory.-Let bosons $b_{1}^{\dagger}=e^{i \phi_{1}}$ be charged under the U(1) symmetry. Then the phase $\phi_{1}$ and conjugate number $n_{1}$ transform as

$$
\begin{aligned}
& \phi_{1} \rightarrow \phi_{1}+\epsilon: \mathrm{U}(1), \quad n_{1} \rightarrow n_{1}: \mathrm{U}(1), \\
& \phi_{1} \rightarrow-\phi_{1}: Z_{2}, \quad n_{1} \rightarrow-n_{1}: Z_{2} .
\end{aligned}
$$

Now we would like to understand how a vortex in a superfluid surface state of this boson field transforms. The remaining $Z_{2}$ symmetry acts on the vortices; however, it is readily seen that this symmetry switches vortices to antivortices. More formally, the vortex fields $\Psi_{2}$ are coupled minimally to the gauge field $\alpha_{2}$, whose flux is the number density $n_{1}=\left(\partial_{x} \alpha_{2 y}-\partial_{y} \alpha_{2 x}\right) / 2 \pi$. Now since the number density changes sign under $Z_{2}$, so does the gauge field $\alpha_{2} \rightarrow-\alpha_{2}$. This relationship implies that, for the minimal coupling to remain invariant, we need $\Psi_{2} \rightarrow \Psi_{2}^{*}$. In fact, the desired transformation is

$$
\Psi_{2} \rightarrow i \sigma_{y} \Psi_{2}^{*}: Z_{2},
$$

which may be viewed as the single projective representation of $\mathrm{U}(1) \rtimes Z_{2}$, where the $\mathrm{U}(1)$ may be viewed as the gauge $\mathrm{U}(1)$ that changes sign under $Z_{2}$. It is readily verified that the gauge-invariant combinations $\left|\psi_{2+}\right|^{2}-\left|\psi_{2-}\right|^{2}$ and $\psi_{2+}^{*} \psi_{2-}=e^{i \phi_{2}}$ both transform nontrivially under $Z_{2}$, verifying that, if vortices condense, they always break the symmetry. A second species of bosons is defined by $b_{2}^{\dagger}=e^{i \phi_{2}}$, which transforms as

$$
\phi_{2} \rightarrow \phi_{2}+\pi: Z_{2}, \quad n_{2} \rightarrow n_{2}: Z_{2}
$$

and is neutral under the global $\mathrm{U}(1)$. This transformation satisfies the intuitive requirements of a topological surface state, and therefore we conclude that a $\mathrm{U}(1) \rtimes Z_{2}$ symmetry group also leads to $Z_{2}$ topological phases. Note, however, if the two symmetries were in direct product, there would be no topological phases.

Let us now write the field theory for the surface in terms of vortices of $\Psi_{2}$. They are minimally coupled to a vector potential $\alpha_{2}$ whose flux is the boson density $\nabla \times \alpha_{1}=n_{1}$.

$$
\mathcal{L}=\left|\left(\partial_{\mu}-i \alpha_{2 \mu}\right) \Psi_{2}\right|^{2}+\frac{1}{2 \kappa} f_{2 \mu \nu}^{2}+\mathcal{V}\left(\left|\Psi_{2}\right|^{2}\right) .
$$

Since the field $b_{1}$ is charged, monopole insertion operators are forbidden, but various anisotropy terms involving four vortex fields are allowed. These and other allowed perturbations are readily identified given the symmetry transformations above.

A dual description of the same theory is obtained by fractionalizing the boson field $b_{1}^{\dagger}=\psi_{1+}^{*} \psi_{1-}$, where $\Psi_{1}=\left(\psi_{1+}, \psi_{1-}\right)$ may be viewed either as a Schwinger boson representation of $b_{1}$ [20] or as vortices of $b_{2}$. Now these transform under a projective representation of the global symmetry $\mathrm{U}(1) \rtimes Z_{2}$ :

$$
\Psi_{1} \rightarrow e^{i \epsilon \sigma_{z} / 2} \Psi_{1}: \mathrm{U}(1),
$$

$$
\Psi_{1} \rightarrow \sigma_{x} \Psi_{1}: Z_{2},
$$

which is compatible with the transformations in Eq. (C1). We see that this implies that vortices in $b_{2}$ carry half unit of global charge at the surface. This information helps us to fix the bulk field theory.

$3 D$ bulk theory.-Given the characterization of the surface states above, we can write a bulk 3D theory that reproduces these features. We write the following theory based on $K=\sigma_{x}$, where the conserved charge is coupled to an external electromagnetic field $A$, and justify it later:

$$
\begin{gathered}
\mathcal{L}_{\text {tot }}=\mathcal{L}_{\text {topo }}+\mathcal{L}_{\text {em }}, \\
\mathcal{L}_{\text {topo }}=\frac{1}{2 \pi} \epsilon\left(B_{1} \partial a_{1}+B_{2} \partial a_{2}\right)+\Theta \frac{\epsilon}{4 \pi^{2}} \partial a_{1} \partial a_{2}, \\
\mathcal{L}_{\text {em }}=\frac{1}{2 \pi} \epsilon\left(B_{1}\right) \partial A .
\end{gathered}
$$

Under the $Z_{2}$ symmetry, $B_{1} \rightarrow-B_{1}, a_{1} \rightarrow-a_{1}$, but $B_{2} \rightarrow B_{2}, a_{2} \rightarrow a_{2}$. Thus the "axion" field $\Theta$ is odd under $Z_{2}$, so the action as a whole is invariant. This observation allows us to fix $\Theta=0, \pi$; the latter value yields the topological phase. Also, only one of the boson species carries global U(1) charge, which implies that there is no topological contribution to the magnetoelectric polarizability, i.e., $\theta=0$.

\section{APPENDIX D: SYMMETRY TRANSFORMATION OF SURFACE STATES WITH TOPOLOGICAL ORDER}

For convenience, we accumulate in this appendix the properties of the surface state with $Z_{2}$ topological order of the SPT phases with various symmetries. As described in the main paper, such a surface topologically ordered phase

\begin{tabular}{|c|c|}
\hline Field & $\overline{\mathcal{T}^{2}}$ \\
\hline$e$ & -1 \\
\hline$m$ & -1 \\
\hline
\end{tabular}
provides a particularly simple perspective on why a trivial gapped symmetry-preserving surface is not allowed. The $Z_{2}$ topological order has four distinct quasiparticles, which we denote as $1, e, m, f$. The trivial quasiparticle sector is described by 1 and consists of all local operators. We take $e$ (for "electric") and $m$ (for "magnetic") to be bosons and $f$ to be a fermion. $e, m$, and $f$ are all mutual semions. Below

TABLE III. $\mathrm{U}(1) \rtimes Z_{2}^{T}$.

\begin{tabular}{llc}
\hline \hline Field & $q$ & $\mathcal{T}^{2}$ \\
\hline$e$ & $\frac{1}{2}$ & 1 \\
$m$ & $\frac{1}{2}$ & 1 \\
\hline \hline
\end{tabular}


TABLE V. $\quad \mathrm{U}(1) \times Z_{2}^{T}$ : Phase 1.

\begin{tabular}{lll}
\hline \hline Field & $q$ & $\mathcal{T}^{2}$ \\
\hline$e$ & $\frac{1}{2}$ & -1 \\
$m$ & 0 & -1 \\
\hline \hline
\end{tabular}

TABLE VI. $\mathrm{U}(1) \times Z_{2}^{T}$ : Phase 2 .

\begin{tabular}{lll}
\hline \hline Field & $q$ & $\mathcal{T}^{2}$ \\
\hline$e$ & $\frac{1}{2}$ & -1 \\
$m$ & $\frac{1}{2}$ & -1 \\
\hline \hline
\end{tabular}

we summarize how the physical symmetry is realized for each of the three nontrivial quasiparticles for the various phases. In what follows, $q$ denotes the charge under the global U(1) symmetry. In all cases, other possible quasiparticles in the same sector are obtained by adding trivial quasiparticles. The symmetry properties of $f$ follow from those of $e$ and $m$ as $f$ is a bound state of $e$ and $m$.

(1) Symmetry $\mathrm{U}(1) \rtimes Z_{2}^{T}$.- - Here both $e$ and $m$ carry charge $1 / 2$ and are time-reversal invariant. It is important only to ask about the presence or absence of Kramers degeneracy under $Z_{2}^{T}$ corresponding to $\mathcal{T}^{2}= \pm 1$. See Table III.

(2) Symmetry $Z_{2}^{T}$.- -Here there are a pair of $e$ particles (denoted $e_{\alpha}=\left(e_{\uparrow}, e_{\downarrow}\right)$, a pair of $m$ particles $\left(m_{\alpha}=\right.$ $\left.\left(m_{\uparrow}, m_{\downarrow}\right)\right)$, and a single $f$ particle. We note that, in the absence of other symmetries, $e_{\uparrow}$ is able to mix with $e_{\downarrow}^{*}$ so that we may regard them as a single particle and its antiparticle, which together form a Kramers doublet. The same reasoning also applies to the $m$ particles. Therefore, we work with just a single $e$ and a single $m$ particle. See Table IV.

(3) Symmetry $\mathrm{U}(1) \times Z_{2}^{T}$.- - Here we discuss two phases, phase 1 and phase 2 . Their symmetries are described in Tables V and VI.

As we emphasized in the main paper, the realization of symmetry is such that these $Z_{2}$ topologically ordered phases cannot arise in strict 2D models with local action of the symmetry group, as is readily seen from the general $K$-matrix classification of $2 \mathrm{D}$ time-reversal-invariant gapped Abelian phases in Ref. [43]. Here we present an elementary analysis that is sufficient for the purposes of this paper. Strictly 2D systems admit an edge to the vacuum (or equivalently a trivial gapped insulator) without changing the symmetry. This is a key difference from the surface topological order of interest to us here, where a domain wall with a trivial gapped insulator is not possible without breaking symmetry. The analysis below, following the reasoning of Ref. [43], relies crucially on analyzing the symmetries of the edge Lagrangian that describes a strictly $2 \mathrm{D}$ system. Hence it distinguishes between topological order that is allowed in strict $2 \mathrm{D}$ and orders that require a
3D bulk. For the case of $Z_{2}$ topological order, we may, as usual, take

$$
K=\left(\begin{array}{ll}
0 & 2 \\
2 & 0
\end{array}\right) .
$$

The corresponding Chern-Simons Lagrangian is simply

$$
\mathcal{L}=\frac{1}{\pi} a_{e} d a_{m}+\frac{1}{2 \pi} A\left(\tau_{e} d a_{e}+\tau_{m} d a_{m}\right)
$$

Here, $a_{1,2}$ are internal gauge fields and $A$ is an external "probe" gauge field. The charge vector $\left(\tau_{e}, \tau_{m}\right)$ has integer components. Physically, $d a_{e, m}$ are $2 \pi j_{e, m}$ of the $e$ and $m$ particles, respectively. If both $e$ and $m$ carry global $\mathrm{U}(1)$ charge $1 / 2$, then $\tau_{e}=\tau_{m}=1$. As we already noted, such a charge assignment implies a nonzero Hall conductivity and hence cannot describe a strict $2 \mathrm{D}$ system with time-reversal invariance. Thus the surface topological order described in Tables III and VI cannot occur in strict 2D systems. It remains for us to discuss the other two surface topological orders. In both (as well as in the case in Table VI) both $e$ and $m$ are Kramers doublets. We now show that this is not possible in any time-reversal-invariant strict $2 \mathrm{D} Z_{2}$ topologically ordered state.

The $1+1$ dimensional edge theory corresponding to the Chern-Simons Lagrangian above is

$$
\mathcal{L}_{\text {edge }}=\frac{1}{\pi} \partial_{t} \phi_{e} \partial_{x} \phi_{m}+\cdots,
$$

with $a_{i 1,2}=\partial_{i} \phi_{1,2}$. Demanding time-reversal invariance of the edge Lagrangian, we see immediately that the edge densities $\partial_{x} \phi_{e}, \partial_{x} \phi_{m}$ must transform with opposite signs. However, if $e^{i \phi_{e}}$ creates one member of a Kramers doublet, it must transform as

$$
e^{i \phi_{e}} \rightarrow i e^{-i \phi_{e}}
$$

under time reversal. Equivalently, $\phi_{e} \rightarrow \phi_{e}+\frac{\pi}{2}$ so that the edge density $\partial_{x} \phi_{e} \rightarrow \partial_{x} \phi_{e}$. Thus if both $e$ and $m$ are Kramers doublets, then both corresponding edge densities must be even under time reversal. But this result is inconsistent with our deduction above from demanding timereversal invariance of the edge Lagrangian. We thus conclude that, in strict 2D systems, both $e$ and $m$ cannot be Kramers pairs in a time-reversal-invariant system. It is allowed to happen, however, at the surface of the 3D SPT phases described in this paper. In passing, we note that this analysis precludes the possibility that strictly $2 \mathrm{D}$ spin models have gapped $Z_{2}$ topological phases where both nontrivial bosonic quasiparticles carry spin- $1 / 2$ (i.e., are spinons) while the fermionic quasiparticle carries no spin.

[1] M.Z. Hasan and C.L. Kane, Colloquium: Topological Insulators, Rev. Mod. Phys. 82, 3045 (2010); X.-L. Qi and S.-C. Zhang, Topological Insulators and 
Superconductors, Rev. Mod. Phys. 83, 1057 (2011); M.Z. Hasan and J.E. Moore, Three-Dimensional Topological Insulators, Annu. Rev. Condens. Matter Phys. 2, 55 (2011).

[2] Andreas P. Schnyder, Shinsei Ryu, Akira Furusaki, Andreas W.W. Ludwig, Classification of Topological Insulators and Superconductors in Three Spatial Dimensions, Phys. Rev. B 78, 195125 (2008); Alexei Kitaev, Periodic Table for Topological Insulators and Superconductors, arXiv:0901.2686.

[3] X. G. Wen, Quantum Field Theory of Many-Body Systems: From the Origin of Sound to an Origin of Light and Electrons (Oxford University Press, Oxford, England, 2004).

[4] This terminology differs slightly from that of Chen et al. [5], who require that, for a state to be short-range entangled, it must also be nonchiral.

[5] Xie Chen, Zheng-Cheng Gu, Zheng-Xin Liu, and XiaoGang Wen, Symmetry Protected Topological Orders and the Group Cohomology of Their Symmetry Group, arXiv:1106.4772.

[6] A. Auerbach, Interacting Electrons and Quantum Magnetism, Graduate Texts in Contemporary Physics (Springer-Verlag, New York, 1994).

[7] L. Fidkowski and A. Kitaev, Topological Phases of Fermions in One Dimension, Phys. Rev. B 83, 075103 (2011).

[8] A. M. Turner, F. Pollmann, and E. Berg, Topological Phases of One-Dimensional Fermions: An Entanglement Point of View, Phys. Rev. B 83, 075102 (2011).

[9] X. Chen, Z.-C. Gu, and X.-G. Wen, Classification of Gapped Symmetric Phases in One-Dimensional Spin Systems, Phys. Rev. B 83, 035107 (2011).

[10] N. Schuch, D. Pérez-Garcia, and I. Cirac, Classifying Quantum Phases Using Matrix Product States and Projected Entangled Pair States, Phys. Rev. B 84, 165139 (2011).

[11] Alexei Kitaev, Anyons in an Exactly Solved Model and Beyond, Ann. Phys. (Amsterdam) 321, 2 (2006); Alexei Kitaev, Toward Topological Classification of Phases with Short-Range Entanglement (unpublished). See http://online .kitp.ucsb.edu/online/topomat11/kitaev/.

[12] M. Levin and Z. Gu, Braiding Statistics Approach to Symmetry-Protected Topological Phases, Phys. Rev. B 86, 115109 (2012).

[13] Yuan-Ming Lu and Ashvin Vishwanath, Theory and Classification of Interacting 'Integer' Topological Phases in Two Dimensions: A Chern-Simons Approach, Phys. Rev. B 86, 125119 (2012).

[14] T. Senthil and Michael Levin, Integer Quantum Hall Effect for Bosons: A Physical Realization, Phys. Rev. Lett. 110, 046801 (2013).

[15] Zheng-Xin Liu and Xiao-Gang Wen, Symmetry-Protected Quantum Spin Hall Phases in Two Dimensions, Phys. Rev. Lett. 110, 067205 (2013).

[16] Xie Chen and Xiao-Gang Wen, Chiral Symmetry on the Edge of Two-Dimensional Symmetry Protected Topological Phases, Phys. Rev. B 86, 235135 (2012).

[17] Xiao-Liang Qi, Taylor L. Hughes, and Shou-Cheng Zhang, Topological Field Theory of Time-Reversal Invariant Insulators, Phys. Rev. B 78, 195424 (2008).
[18] Andrew M. Essin, Joel E. Moore, and David Vanderbilt, Magnetoelectric Polarizability and Axion Electrodynamics in Crystalline Insulators, Phys. Rev. Lett. 102, 146805 (2009).

[19] T. Senthil and M.P.A. Fisher, $Z_{2}$ Gauge Theory of Electron Fractionalization in Strongly Correlated Systems, Phys. Rev. B 62, 7850 (2000).

[20] T. Senthil, A. Vishwanath, Leon Balents, Subir Sachdev, and M. P. A. Fisher, Deconfined Quantum Critical Points, Science 303, 1490 (2004); T. Senthil, Leon Balents, Subir Sachdev, Ashvin Vishwanath, and Matthew P. A. Fisher, Quantum Criticality beyond the Landau-Ginzburg-Wilson Paradigm, Phys. Rev. B 70, 144407 (2004).

[21] D. Birmingham, M. Blau, M. Rakowski, and G. Thompson, Topological Field Theory, Phys. Rep. 209, 129 (1991).

[22] T.H. Hansson, V. Oganesyan, and S. Sondhi, Superconductors Are Topologically Ordered, Ann. Phys. (Amsterdam) 313, 497 (2004).

[23] Kevin Walker and Zhenghan Wang, $(3+1)-T Q F T s$ and Topological Insulators, arXiv:1104.2632.

[24] C. W. von Keyserlingk, F. J. Burnell, and Steven H. Simon, Three-Dimensional Topological Lattice Models with Surface Anyons, Phys. Rev. B 87, 045107 (2013).

[25] G. Y. Cho and J. E. Moore, Topological BF Field Theory Description of Topological Insulators, Ann. Phys. (Amsterdam) 326, 1515 (2011).

[26] H. Aratyn, Fermions from Bosons in $2+1$ Dimensions, Phys. Rev. D 28, 2016 (1983).

[27] AtMa Chan, T. Hughes, S. Ryu, and E. Fradkin, Effective Field Theories for Topological Insulators by Functional Bosonization, arXiv:1210.4305.

[28] Masaki Oshikawa, Commensurability, Excitation Gap, and Topology in Quantum Many-Particle Systems on a Periodic Lattice, Phys. Rev. Lett. 84, 1535 (2000).

[29] M. B. Hastings, Lieb-Schultz-Mattis in Higher Dimensions, Phys. Rev. B 69, 104431 (2004).

[30] C. Lannert, Matthew P. A. Fisher, and T. Senthil, Quantum Confinement Transition in a $d$-Wave Superconductor, Phys. Rev. B 63, 134510 (2001).

[31] Scott D. Geraedts and Olexei I. Motrunich, Monte Carlo Study of a $U(1) \times U(1)$ System with $\pi$-Statistical Interaction, Phys. Rev. B 85, 045114 (2012).

[32] Andreas W. W. Ludwig, Matthew P. A. Fisher, R. Shankar, and G. Grinstein, Integer Quantum Hall Transition: An Alternative Approach and Exact Results, Phys. Rev. B 50, 7526 (1994).

[33] T. Senthil and Matthew P. A. Fisher, Competing Orders, Nonlinear Sigma Models, and Topological Terms in Quantum Magnets, Phys. Rev. B 74, 064405 (2006).

[34] B. Swingle, M. Barkeshli, J. McGreevy, and T. Senthil, Correlated Topological Insulators and the Fractional Magnetoelectric Effect, Phys. Rev. B 83, 195139 (2011).

[35] Michael Levin, F. J. Burnell, Maciej Koch-Janusz, and Ady Stern, Exactly Soluble Models for Fractional Topological Insulators in Two and Three Dimensions, Phys. Rev. B 84, 235145 (2011).

[36] Joseph Maciejko, Xiao-Liang Qi, Andreas Karch, and Shou-Cheng Zhang, Fractional Topological Insulators in Three Dimensions, Phys. Rev. Lett. 105, 246809 (2010). 
[37] J. T. Chalker and P. D. Coddington, Percolation, Quantum Tunnelling and the Integer Hall Effect, J. Phys. C 21, 2665 (1988).

[38] C. Dasgupta and B.I. Halperin, Phase Transition in a Lattice Model of Superconductivity, Phys. Rev. Lett. 47, 1556 (1981).

[39] M.P. A. Fisher and D. H. Lee, Correspondence between Two-Dimensional Bosons and a Bulk Superconductor in a Magnetic Field, Phys. Rev. B 39, 2756 (1989).

[40] Leon Balents, Lorenz Bartosch, Anton Burkov, Subir Sachdev, and Krishnendu Sengupta, Putting Competing Orders in Their Place near the Mott Transition, Phys. Rev. B 71, 144508 (2005).

[41] Olexei I. Motrunich and Ashvin Vishwanath, Emergent Photons and Transitions in the O(3) Sigma Model with Hedgehog Suppression, Phys. Rev. B 70, 075104 (2004).

[42] Cenke Xu and A. W. W. Ludwig, Nonperturbative Effects of Topological Theta-Term, arXiv:1112.5303.

[43] Michael Levin and Ady Stern, Classification and Analysis of Two-Dimensional Abelian Fractional Topological Insulators, Phys. Rev. B 86, 115131 (2012).

[44] With an integer coefficient $Q, \mathcal{L}_{b}^{E}=\frac{Q}{2 \pi} \epsilon B \partial a$ describes a topologically ordered phase with ground-state degeneracy of $Q^{3}$ on a three torus.

[45] This is obtained by applying Gauss's law $\int d V \partial_{\mu} j^{\mu}=$ $\oint d S_{\mu} j^{\mu}$, where $j^{\mu}=\epsilon^{\mu \nu \lambda \sigma} a_{1 \nu} \partial_{\lambda} a_{2 \sigma}$.

[46] O. I. Motrunich and T. Senthil, Origin of Artificial Electrodynamics in Three-Dimensional Bosonic Models, Phys. Rev. B 71, 125102 (2005).

[47] O. I. Motrunich and A. Vishwanath, Comparative Study of Higgs Transition in One-Component and Two-Component Lattice Superconductor Models, arXiv:0805.1494.

[48] A. B. Kuklov, M. Matsumoto, N. V. Prokofev, B. V. Svistunov, and M. Troyer, Deconfined Criticality: Generic First-Order Transition in the SU(2) Symmetry Case, Phys. Rev. Lett. 101, 050405 (2008).

[49] F. Jiang, M. Nyfeler, S. Chandrasekharan, and U. Wiese, From an Antiferromagnet to a Valence Bond Solid: Evidence for a First-Order Phase Transition, J. Stat. Mech. (2008) P02009.
[50] A.W. Sandvik, Evidence for Deconfined Quantum Criticality in a Two-Dimensional Heisenberg Model with Four-Spin Interactions, Phys. Rev. Lett. 98, 227202 (2007); Anders W. Sandvik, Continuous Quantum Phase Transition between an Antiferromagnet and a ValenceBond Solid in Two Dimensions: Evidence for Logarithmic Corrections to Scaling, Phys. Rev. Lett. 104, 177201 (2010).

[51] A. Banerjee, K. Damle, and F. Alet, Impurity Spin Texture at a Deconfined Quantum Critical Point, Phys. Rev. B 82, 155139 (2010).

[52] R. G. Melko and R. K. Kaul, Scaling in the Fan of an Unconventional Quantum Critical Point, Phys. Rev. Lett. 100, 017203 (2008); Ribhu K. Kaul and Anders W. Sandvik, Lattice Model for the $S U(N)$ Néel to ValenceBond Solid Quantum Phase Transition at Large N, Phys. Rev. Lett. 108, 137201 (2012).

[53] Anatoly Kuklov, Nikolay Prokof'ev, and Boris Svistunov, Weak First-Order Superfluid-Solid Quantum Phase Transitions, Phys. Rev. Lett. 93, 230402 (2004).

[54] J. T. Chayes, L. Chayes, Daniel S. Fisher, and T. Spencer, Finite-Size Scaling and Correlation Lengths for Disordered Systems, Phys. Rev. Lett. 57, 2999 (1986).

[55] P. M. Ostrovsky, I. V. Gornyi, and A.D. Mirlin, Interaction-Induced Criticality in $\mathrm{Z}_{2}$ Topological Insulators, Phys. Rev. Lett. 105, 036803 (2010).

[56] Victor M. Galitski, G. Refael, Matthew P. A. Fisher, and T. Senthil, Vortices and Quasiparticles near the Superconductor-Insulator Transition in Thin Films, Phys. Rev. Lett. 95, 077002 (2005).

[57] Matthew Fisher (personal communication).

[58] B. Swingle, Interplay between Short and Long-Range Entanglement in Symmetry Protected Phases, arXiv:1209.0776.

[59] Cenke Xu, Three Dimensional Symmetry Protected Topological Phase Close to Antiferromagnetic Neel Order, arXiv:1209.4399. 